\title{
Funnel control of nonlinear systems
}

\author{
Thomas Berger $^{1}$ (D) Achim Ilchmann ${ }^{2}$. Eugene P. Ryan ${ }^{3}$
}

Received: 20 July 2020 / Accepted: 16 January 2021 / Published online: 26 February 2021

(C) The Author(s) 2021

\begin{abstract}
Tracking of reference signals is addressed in the context of a class of nonlinear controlled systems modelled by $r$-th-order functional differential equations, encompassing inter alia systems with unknown "control direction" and dead-zone input effects. A control structure is developed which ensures that, for every member of the underlying system class and every admissible reference signal, the tracking error evolves in a prescribed funnel chosen to reflect transient and asymptotic accuracy objectives. Two fundamental properties underpin the system class: bounded-input bounded-output stable internal dynamics, and a high-gain property (an antecedent of which is the concept of sign-definite high-frequency gain in the context of linear systems).
\end{abstract}

Keywords Nonlinear systems - Adaptive control - Asymptotic tracking · Funnel control $\cdot$ Relative degree $\cdot$ Functional differential equations

Mathematics Subject Classification 93C10 $\cdot 93 \mathrm{C} 23 \cdot 93 \mathrm{C} 40$

\section{Nomenclature}

$\mathbb{N}, \mathbb{N}_{0} \quad$ The set of positive, non-negative integers, respectively

$\mathbb{R}_{\geq 0}, \mathbb{C}_{\geq 0} \quad$ The sets $[0, \infty),\{\lambda \in \mathbb{C} \mid \operatorname{Re}(\lambda) \geq 0\}$, respectively

This work was supported by the German Research Foundation (Deutsche Forschungsgemeinschaft) via the Grant BE 6263/1-1.

$凶$ Thomas Berger

thomas.berger@math.upb.de

Achim Ilchmann

achim.ilchmann@tu-ilmenau.de

Eugene P. Ryan

masepr@bath.ac.uk

1 Institut für Mathematik, Universität Paderborn, Warburger Str. 100, 33098 Paderborn, Germany

2 Institut für Mathematik, Technische Universität Ilmenau, Weimarer Straße 25, 98693 Ilmenau, Germany

3 Department of Mathematical Sciences, University of Bath, Bath BA2 7AY, UK 


$\begin{aligned}\langle v, w\rangle & \text { The Euclidean inner product of vectors } v, w \in \mathbb{R}^{n} \\ \|x\| & \sqrt{\langle x, x\rangle} \text {, the Euclidean norm of } x \in \mathbb{R}^{n} \\ L^{\infty}\left(I, \mathbb{R}^{n}\right) & \text { The Lebesgue space of measurable, essentially bounded functions } \\ & f: I \rightarrow \mathbb{R}^{n} \text {, where } I \subseteq \mathbb{R} \text { is some interval } \\ L_{\text {loc }}^{\infty}\left(I, \mathbb{R}^{n}\right) & \text { The set of measurable, locally essentially bounded functions } \\ & f: I \rightarrow \mathbb{R}^{n}, \text { where } I \subseteq \mathbb{R} \text { is some interval } \\ W^{k, \infty}\left(I, \mathbb{R}^{n}\right) & \text { The Sobolev space of all functions } f: I \rightarrow \mathbb{R}^{n} \text { with } k \text {-th-order } \\ & \text { weak derivative } f^{(k)} \text { and } f, f^{(1)}, \ldots, f^{(k)} \in L^{\infty}\left(I, \mathbb{R}^{n}\right), \text { where } \\ & I \subseteq \mathbb{R} \text { is some interval and } k \in \mathbb{N} \\ C^{k}\left(V, \mathbb{R}^{n}\right) & \text { The set of } k \text {-times continuously differentiable functions } f: V \rightarrow \\ & \mathbb{R}^{n}, \text { where } V \subseteq \mathbb{R}^{m} \text { and } k \in \mathbb{N}_{0} ; C\left(V, \mathbb{R}^{n}\right):=C^{0}\left(V, \mathbb{R}^{n}\right)\end{aligned}$

\section{Introduction}

Since its inception in 2002, the concept of funnel control has been widely investigated. In its essence, the approach considers the following basic question: for a given class of dynamical systems, with input $u$ and output $y$, and a given class of reference signals $y_{\text {ref }}$, does there exist a single control strategy (generating $u$ ) which ensures that, for every member of the system class and every admissible reference signal, the output $y$ approaches the reference $y_{\text {ref }}$ with prescribed transient behaviour and prescribed asymptotic accuracy? The twofold objective of "prescribed transient behaviour and asymptotic accuracy" is encompassed by the adoption of a so-called "performance funnel" in which the error function $t \mapsto e(t):=y(t)-y_{\mathrm{ref}}(t)$ is required to evolve; see Fig. 1. Underlying the present paper is a large class of systems described by $r$-th-order functional differential equations: we denote this class (which will be made precise in due course) by $\mathscr{N}^{m, r}$, where $m \in \mathbb{N}$ denotes the dimension of both input and output. The information available for feedback to the controller is comprised of the instantaneous values of the output and its first $r-1$ derivatives, together with the instantaneous values of the reference signal and its first $\hat{r}-1$ derivatives, where $1 \leq \hat{r} \leq r$. A feedback strategy is developed which assures attainment of the above twofold performance objective: this is the core of the main result, Theorem 1.9. We proceed to highlight the features and distinguishing novelties of this result vis à vis the existing literature.

\subsection{Novelties and literature}

\section{Predecessors and relative degree}

The parameter $r$ coincides with the concept of relative degree for many nonlinear examples belonging to the class $\mathscr{N}^{m, r}$. The class is, however, of sufficient generality to encompass, not only such examples, but also systems which do not necessarily have a relative degree as defined in, for example, [35]. Adaptive control for systems with relative degree $r>1$ has been an issue since the early days of high-gain adaptive control, as evidenced by the contribution [40] from 1984. An early approach which takes transient behaviour into account is [41] in 1991, using a feedback strategy that differs 
conceptually from the funnel control methodology. Funnel control was introduced in 2002 by [30] for nonlinear functional systems of the form (1) with relative degree one, using a variant of the high-gain property from Definition 1.2. The efficacy of funnel control for systems (1) with arbitrary $r \in \mathbb{N}$ was demonstrated in [32] in 2007. However, the control structure in that paper is based on backstepping with attendant (but unavoidable) escalating controller complexity vis à vis the striking simplicity of the funnel controller for relative-degree-one systems. An alternative controller was developed in [38] for a special class of systems with $m=1$ and arbitrary $r \in \mathbb{N}$, termed the bang-bang funnel controller. Since the control input switches only between two values, it is able to respect input constraints; however, it requires various feasibility assumptions and involves a complicated switching logic. A simpler control strategy for nonlinear system has been introduced by [25] for $r=2$ in 2013 and by [8] for $r \in \mathbb{N}$ in 2018.

\section{Controller complexity}

Already alluded to in the above paragraph, some explicit remarks on the issue of controller complexity may be warranted. For implementation purposes, the avoidance of excessive complexity is crucial. The first approaches to funnel control for systems with arbitrary relative degree in $[31,32]$ showed a significant increase in controller complexity with increasing relative degree (a variant of the "curse of dimensionality"). Although these contributions have the advantage that only the output-and not its derivatives - need to be known, they involve an intrinsic backstepping procedure which requires increasing powers of a particular gain function as the relative degree grows. For "large" relative degree, this leads to impracticality. Avoiding the backstepping procedure, a low-complexity funnel controller has been developed in [25] for relative degree two systems and in [8] for arbitrary relative degree. Nevertheless, the control design developed in [8] involves successive derivatives of particular auxiliary error variables, causing high-level complexity for high relative degree. The relative simplicity of funnel control design underpinning Theorem 1.9 helps to resolve these complexity issues.

\section{Unknown control direction}

In the early days of high-gain adaptive control without system identification, linear systems with relative degree one and positive high-frequency gain were studied, cf. Sect. 2.1. In 1983, Morse [42] conjectured the non-existence of a smooth adaptive controller which stabilizes every such system under the weakened assumption that the high-frequency gain is not zero, but its sign is unknown. Nussbaum [44] showed (constructively) that Morse's conjecture is false. He introduced a class of sign-sensing or probing "switching functions" in the feedback design, see Sect. 2.2. In the present work, we allow for a larger class of switching functions (namely, continuous surjective maps $[0, \infty) \rightarrow \mathbb{R}$, which properly contain the "Nussbaum" class), potentially advantageous in applications.

\section{Dead-zone input}

A dead-zone input is a special case of input nonlinearity where the value of the nonlinearity is zero when the input is between some prescribed deadband parameters, see Sects. 2.3 and 2.4. A dead-zone input may appear in practical applications such as 
hydraulic servo valves and electronic motors, and it may severely affect the performance of a control system, see e.g. [55,56]. Several approaches have been undertaken to treat these problems, see $[43,55,56]$ and the references therein. We show that the system class $\mathscr{N}^{m, r}$ encompasses a larger class of dead-zone inputs than previously considered in the literature.

\section{Practical and exact asymptotic tracking}

The "performance funnel", which we denote by

$$
\mathscr{F}_{\varphi}:=\left\{(t, e) \in \mathbb{R}_{\geq 0} \times \mathbb{R}^{m} \mid \varphi(t)\|e\|<1\right\},
$$

in which the tracking error is required to evolve, is determined by the choice of a continuous function $\varphi: \mathbb{R}_{\geq 0} \rightarrow \mathbb{R}_{\geq 0}$ with requisite properties which include positivity on $(0, \infty)$ and boundedness away from zero "at infinity":

$$
\forall t>0: \varphi(t)>0 \text { and } \liminf _{t \rightarrow \infty} \varphi(t)>0 .
$$

For example, the unbounded function $\varphi: t \mapsto e^{\alpha t}-1, \alpha>0$, is an admissible choice, in which case evolution in $\mathscr{F}_{\varphi}$ ensures that the tracking error $e(\cdot)$ converges to zero exponentially fast. In particular, exact asymptotic tracking is achieved. However, there is a price to pay. A fundamental ingredient of the funnel controller is the quantity $\varphi(t) e(t)$ which, in the case of unbounded $\varphi$, inevitably leads to an ill-conditioned computation of the product of "infinitely large" and "infinitesimally small" terms. Therefore, while of theoretical interest, the case of unbounded $\varphi$ may be of limited utility in applications. If $\varphi$ is bounded, then the radius of the funnel $t$-section $\mathscr{F}_{\varphi} \cap\left(\{t\} \times \mathbb{R}^{m}\right)$ is uniformly bounded away from zero and so asymptotic tracking is not achieved. However, the choice of (bounded) $\varphi$ is at the designer's discretion and so practical tracking with arbitrarily small (but non-zero) prescribed asymptotic accuracy is achievable without encountering the ill-conditioning present in the exact asymptotic tracking case.

The assumption of bounded $\varphi$ is widespread in the literature on funnel control. Exact asymptotic tracking with unbounded $\varphi$ was achieved in [50] for a class of nonlinear relative degree one systems: in [27] a predecessor for linear relative degree one systems was developed utilizing the internal model principle. Recently (and unaware of the latter results) it was observed in [37] that asymptotic funnel control is possible for a class of nonlinear single-input single-output systems, albeit more restrictive than the class $\mathscr{N}^{m, r}$ of the present paper. Note also that asymptotic tracking via funnel control for systems with relative degree two has been shown by $[59,60]$. However, the radius of the funnel in these works is bounded away from zero and the property of exact asymptotic tracking is achieved at the expense of a discontinuous control scheme.

\section{Parameter $\hat{r} \leq r$}

Throughout, it is assumed that the instantaneous values of the output $y(t)$ and its first $r-1$ derivatives, together with the instantaneous value $y_{\mathrm{ref}}(t)$ of the reference signal, are available for feedback purposes. However, in applications, some derivatives of the reference signal may not be accessible by the controller. The parameter 
$\hat{r} \in\{1, \ldots, r\}$ quantifies the number of derivatives that are available, and so the instantaneous information signal fed to the controller is encapsulated by the vector $\mathbf{e}(t)=\left(e(t), \ldots, e^{(\hat{r}-1)}, y^{(\hat{r})}(t), \ldots, y^{(r-1)}(t)\right)$ with $e(t)=y(t)-y_{\text {ref }}(t)$. The potential to cope with non-availability of reference signal derivatives might be advantageous for applications. Of course, the larger the value of $\hat{r}$, the more information is available for control use, and so it might reasonably be expected that controller "behaviour" improves with increasing $\hat{r}$. This expectation is borne out by numerical simulations.

\section{Prescribed performance control}

A relative of funnel control is the approach of prescribed performance control developed by Bechlioulis and Rovithakis [1] in 2008. Using so-called performance functions (which admit a funnel interpretation) and a transformation that incorporates these functions, the original controlled system is expressed in a form for which boundedness of the states, via the prescribed performance control input, can be proved-achieving evolution of the tracking error within the funnel defined by the performance functions. The controller presented in [1] is not of high-gain type. Instead, neural networks are used to approximate the unknown nonlinearities of the system, resulting in a complicated controller structure. After some developments, the complexity issue has been addressed in [2] in 2014, where prescribed performance control is shown to be feasible for systems in pure feedback for. However, the $t$-sections of the funnels corresponding to the underlying performance functions have radii bounded away from zero and so exact asymptotic tracking cannot be achieved, see e.g. [2]. While funnel control and prescribed performance control are motivated by similar design objectives, the solution methodologies are intrinsically different.

\section{Applications}

The new funnel control strategy has a potential impact on various applications. Since its development in [30], the funnel controller proved an appropriate tool for tracking problems in various applications such as temperature control of chemical reactor models [33], control of industrial servo-systems [24] and underactuated multibody systems [9], speed control of wind turbine systems [21,23], current control for synchronous machines [22], DC-link power flow control [52], voltage and current control of electrical circuits [14], oxygenation control during artificial ventilation therapy [45], control of peak inspiratory pressure [46] and adaptive cruise control $[12,13]$.

\subsection{System class}

We make precise the underlying class $\mathscr{N}^{m, r}$ of systems, modelled by nonlinear functional differential equations of the form

$$
\begin{aligned}
y^{(r)}(t) & =f\left(d(t), \mathbf{T}\left(y, \dot{y}, \ldots, y^{(r-1)}\right)(t), u(t)\right) \\
\left.y\right|_{[-h, 0]} & =y^{0} \in C^{r-1}\left([-h, 0], \mathbb{R}^{m}\right),
\end{aligned}
$$

where $h \geq 0$ quantifies the "memory" in the system, $r \in \mathbb{N}$ is related to the concept of relative degree, $m \in \mathbb{N}$ is the dimension of both the input $u(t)$ and output $y(t)$ at time $t \geq 0, d \in L^{\infty}\left(\mathbb{R}_{\geq 0}, \mathbb{R}^{p}\right)$ is a "disturbance", and $f \in C\left(\mathbb{R}^{p} \times \mathbb{R}^{q} \times \mathbb{R}^{m}, \mathbb{R}^{m}\right)$ 
belongs to a set of nonlinear functions characterized by a particular a high-gain property (made precise in Definition 1.2). The operator $\mathbf{T}: C\left([-h, \infty), \mathbb{R}^{n}\right) \rightarrow$ $L_{\text {loc }}^{\infty}\left(\mathbb{R}_{\geq 0}, \mathbb{R}^{q}\right)$, where $n=r m$, belongs to the class $\mathbb{T}_{h}^{n, q}$ of mappings which are causal, satisfy a local Lipschitz condition, and map bounded functions to bounded functions (made precise in Definition 1.1). The most simple, but non-trivial, prototype of the system class $\mathscr{N}^{m, r}$ is linear systems with strict relative degree $r$ and asymptotically stable zero dynamics (see Sect. 2.1).

Definition 1.1 (Operator class) For $n, q \in \mathbb{N}$ and $h \geq 0$, the set $\mathbb{T}_{h}^{n, q}$ denotes the class of operators

$$
\mathbb{T}_{h}^{n, q}:=\left\{\mathbf{T}: C\left([-h, \infty), \mathbb{R}^{n}\right) \rightarrow L_{\mathrm{loc}}^{\infty}\left(\mathbb{R}_{\geq 0}, \mathbb{R}^{q}\right) \mid(\mathrm{TP} 1)-(\mathrm{TP} 3) \text { hold }\right\},
$$

where (TP1)-(TP3) denote the following properties.

(TP1) Causality: for all $\zeta, \theta \in C\left([-h, \infty), \mathbb{R}^{n}\right)$ and all $t \geq 0$,

$$
\left.\zeta\right|_{[-h, t]}=\left.\left.\theta\right|_{[-h, t]} \Longrightarrow \mathbf{T}(\zeta)\right|_{[0, t]}=\left.\mathbf{T}(\theta)\right|_{[0, t]} .
$$

(TP2) Local Lipschitz property: for each $t \geq 0$ and all $\xi \in C\left([-h, t], \mathbb{R}^{n}\right)$, there exist positive constants $c_{0}, \delta, \tau>0$ such that, for all $\zeta_{1}, \zeta_{2} \in C\left([-h, \infty), \mathbb{R}^{n}\right)$ with $\left.\zeta_{i}\right|_{[-h, t]}=\xi$ and $\left\|\zeta_{i}(s)-\xi(t)\right\|<\delta$ for all $s \in[t, t+\tau]$ and $i=1,2$, we have

$$
\operatorname{ess~sup}_{s \in[t, t+\tau]}\left\|\mathbf{T}\left(\zeta_{1}\right)(s)-\mathbf{T}\left(\zeta_{2}\right)(s)\right\| \leq c_{0} \sup _{s \in[t, t+\tau]}\left\|\zeta_{1}(s)-\zeta_{2}(s)\right\|
$$

(TP3) Bounded-input bounded-output (BIBO) property: for each $c_{1}>0$ there exists $c_{2}>0$ such that, for all $\zeta \in C\left([-h, \infty), \mathbb{R}^{n}\right)$,

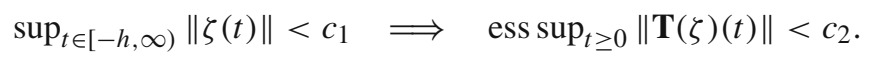

Property (TP1) is entirely natural in the context of physically motivated controlled systems. Property (TP2) is a technical condition which (in conjunction with continuity of $f$ ) plays a role in ensuring well-posedness of the initial-value problem (1) under feedback control. Property (TP3) is, loosely speaking, a stability condition on the "internal dynamics" of (1). For linear systems with strict relative degree, the first two conditions are trivially satisfied, while the third is equivalent to a minimum-phase assumption: this is shown in Sect. 2.1.2.

The formulation also embraces nonlinear delay elements and hysteretic effects, as we shall briefly illustrate.

\section{Nonlinear delay elements.}

For $i=0, \ldots, k$, let $\Psi_{i}: \mathbb{R} \times \mathbb{R}^{m} \rightarrow \mathbb{R}^{q}$ be measurable in its first argument and locally Lipschitz in its second argument, uniformly with respect to its first argument. Precisely, for each $\xi \in \mathbb{R}^{m}, \Psi_{i}(\cdot, \xi)$ is measurable, and for every compact $C \subset \mathbb{R}^{m}$, there exists a constant $c>0$ such that

$$
\text { for a.a. } t \in \mathbb{R} \forall \xi_{1}, \xi_{2} \in C:\left\|\Psi_{i}\left(t, \xi_{1}\right)-\Psi_{i}\left(t, \xi_{2}\right)\right\| \leq c\left\|\xi_{1}-\xi_{2}\right\|
$$


Let $h_{i}>0, i=0, \ldots, k$, and set $h:=\max _{i} h_{i}$. For $y \in C\left([-h, \infty), \mathbb{R}^{m}\right)$, let

$$
\mathbf{T}(y)(t):=\int_{-h_{0}}^{0} \Psi_{0}(s, y(t+s)) \mathrm{d} s+\sum_{i=1}^{k} \Psi_{i}\left(t, y\left(t-h_{i}\right)\right), t \geq 0 .
$$

The operator T, so defined (which models distributed and point delays), is of class $\mathbb{T}_{h}^{m, q}$; for details, see [49].

\section{Hysteresis}

A large class of nonlinear operators $\mathbf{T}: C\left(\mathbb{R}_{\geq 0}, \mathbb{R}\right) \rightarrow C\left(\mathbb{R}_{\geq 0}, \mathbb{R}\right)$, which includes many physically motivated hysteretic effects, is defined in [39]. These operators are contained in the class $\mathbb{T}_{0}^{1,1}$. Specific examples include relay hysteresis, backlash hysteresis, elastic-plastic hysteresis, and Preisach operators. For further details, see [29].

Next, we introduce a high-gain property which, in effect, characterizes the class of admissible nonlinearities $f$.

Definition 1.2 (High-gain property) For $p, q, m \in \mathbb{N}$, a function $f \in C\left(\mathbb{R}^{p} \times \mathbb{R}^{q} \times\right.$ $\left.\mathbb{R}^{m}, \mathbb{R}^{m}\right)$ is said to have the high-gain property, if there exists $v^{*} \in(0,1)$ such that, for every compact $K_{p} \subset \mathbb{R}^{p}$ and compact $K_{q} \subset \mathbb{R}^{q}$, the (continuous) function

$$
\begin{aligned}
\chi: & \mathbb{R} \rightarrow \mathbb{R}, \\
s & \mapsto \min \left\{\langle v, f(\delta, z,-s v)\rangle \mid(\delta, z) \in K_{p} \times K_{q}, v \in \mathbb{R}^{m}, v^{*} \leq\|v\| \leq 1\right\}
\end{aligned}
$$

is such that $\sup _{s \in \mathbb{R}} \chi(s)=\infty$.

We elucidate the high-gain property_which at first sight might seem somewhat arcane-in the following two remarks, the first of which treats the linear case.

Remark 1.3 Why the terminology "high-gain property" and how is it related to "high-gain stabilization"? Consider a very specific class of linear systems with no disturbance $d(\cdot)$ :

$$
\dot{y}(t)=L_{1} y(t)+L_{2} u(t), \quad \text { for } \quad L_{1}, L_{2} \in \mathbb{R}^{m \times m} .
$$

For this system class, the following implications hold.

System (2) has the high-gain property.

$$
L_{2} \in \mathbb{R}^{m \times m} \stackrel{\mathbb{1}}{\text { is sign definite, }}
$$

i.e. there exists $\sigma \in\{-1,1\}$ such that $\sigma\left\langle v, L_{2} v\right\rangle>0$ for all $v \in \mathbb{R}^{m} \backslash\{0\}$.

$$
\Downarrow
$$

System (2) is high-gain stabilizable,

i.e. there exist $\sigma \in\{-1,1\}$ and $k^{*}>0$ such that, for all $k \geq k^{*}$, the control $u(t)=-\sigma k y(t)$ renders system (2) exponentially stable. 
The equivalence of the first two statements is shown in Sect. 2.1.3. If $L_{2} \in \mathbb{R}^{m \times m}$ is sign definite, then there exists $\sigma \in\{-1,1\}$ such that $\sigma L_{2}$ is positive definite and so

$$
\exists k^{*}>0 \forall k \geq k^{*} \forall \lambda \in \mathbb{C}: \operatorname{det}\left(\lambda I_{m}-k^{-1} L_{1}+\sigma L_{2}\right)=0 \Longrightarrow \operatorname{Re} \lambda<0,
$$

whence the conclusion that, for a sufficiently high value of the gain parameter $k>0$, the linear control $u(t)=-\sigma k y(t)$ renders the system exponentially stable. The reverse implication does not hold. As a counterexample, consider (2) with

$$
L_{1}=\left[\begin{array}{cc}
0 & -1 \\
1 & 0
\end{array}\right] \quad \text { and } \quad L_{2}=\left[\begin{array}{ll}
1 & 0 \\
0 & 0
\end{array}\right] \text {, }
$$

which, under the control $u(t)=-k y(t)$, takes the form

$$
\dot{y}(t)=\left(L_{1}-k L_{2}\right) y(t)=\left[\begin{array}{cc}
-k & -1 \\
1 & 0
\end{array}\right] y(t),
$$

which is exponentially stable for all $k>0$ and so (2) is high-gain stabilizable. However, $L_{2}$ is not sign definite.

The high-gain property in Definition 1.2 extrapolates the above observations to a nonlinear setting.

\section{Remark 1.4}

(a) The high-gain property holds for $f \in C\left(\mathbb{R}^{p} \times \mathbb{R}^{q} \times \mathbb{R}^{m}, \mathbb{R}^{m}\right)$ if, and only if, there exists $v^{*} \in(0,1)$ such that, for every compact $K_{p} \subset \mathbb{R}^{p}$ and compact $K_{q} \subset \mathbb{R}^{q}$, at least one of the following two properties is true for the continuous function $\chi$ defined as in Definition 1.2:

$$
\text { (i) } \sup _{s>0} \chi(s)=\infty \text { or (ii) } \sup _{s<0} \chi(s)=\infty \text {. }
$$

If (i) (respectively, (ii)) holds for every such pair $\left(K_{p}, K_{q}\right)$, then we say that $f$ has the negative-definite high-gain property (respectively, the positive-definite high-gain property).

(b) That a function $f$ may have both the negative-definite and positive-definite highgain properties is illustrated by following example. Let $m=1$ and let $f$ (with trivial $(\delta, z)$ dependence) be given by

$$
f(\delta, z, u)=u \sin (\ln (1+|u|)), \quad(\delta, z, u) \in \mathbb{R}^{p} \times \mathbb{R}^{q} \times \mathbb{R},
$$

which has the set of zeros $\left\{u_{k},-u_{k}\right\}$ with

$$
u_{k}=e^{k \pi}-1, \quad k \in \mathbb{N}_{0}
$$

Define the sequence $\left(s_{k}\right)$ by

$$
s_{k}:=\frac{1}{2}\left(u_{k+1}-u_{k}\right)=\frac{1}{2} e^{k \pi}\left(e^{\pi}-1\right)>0, \quad k \in \mathbb{N} .
$$


Noting that $4 e^{\pi / 2}<e^{\pi}-1$, we have

$$
\ln \left(1+\frac{1}{2} s_{k}\right)=\ln \left(e^{k \pi}\left(e^{-k \pi}+\frac{1}{4}\left(e^{\pi}-1\right)\right)\right)>k \pi+\frac{\pi}{2} .
$$

Also,

$\ln \left(1+s_{k}\right)=\ln \left(e^{k \pi}\left(e^{-k \pi}+\frac{1}{2}\left(e^{\pi}-1\right)\right)\right)<\ln \left(e^{k \pi}\left(e^{\pi} / 2\right)\right)=(k+1) \pi-\ln 2$.

Therefore, for all $v \in \mathbb{R}$ with $\frac{1}{2} \leq|v| \leq 1$ we have

$$
k \pi+\frac{\pi}{2}<\ln \left(1+s_{k}|v|\right)<(k+1) \pi-\ln 2 .
$$

It follows that

$$
0<\sin (\pi-\ln 2)<\left\{\begin{array}{l}
+\sin \left(\ln \left(1+s_{k}|v|\right)\right), k \text { even } \\
-\sin \left(\ln \left(1+s_{k}|v|\right)\right), k \text { odd }
\end{array}\right\}<1
$$

Set $v^{*}=\frac{1}{2}$. Then, we find that

$$
\chi\left(-s_{2 k}\right)=\min _{\frac{1}{2} \leq|v| \leq 1} s_{2 k} v^{2} \sin \left(\ln \left(1+s_{2 k}|v|\right)\right)>\frac{1}{4} s_{2 k} \sin (\pi-\ln 2)
$$

and

$\chi\left(s_{2 k+1}\right)=\min _{\frac{1}{2} \leq|v| \leq 1}-s_{2 k+1} v^{2} \sin \left(\ln \left(1+s_{2 k+1}|v|\right)\right)>\frac{1}{4} s_{2 k+1} \sin (\pi-\ln 2)$.

Since $\sin (\pi-\ln 2)>0$, it follows that $\sup _{s>0} \chi(s)=\infty=\sup _{s<0} \chi(s)$. Therefore, $f$ has both the negative-definite and positive-definite high-gain properties.

(c) For linear systems with strict relative degree, we show in Sect. 2.1.3 that (i) (respectively, (ii)) is equivalent to the high-frequency gain matrix being negative definite (respectively, positive definite).

(d) If it is known in advance that the negative-definite (respectively, positivedefinite) high-gain property holds; then, the controller structure can be simplified considerably as we will discuss in Remark 1.8.

Now we are in a position to define the general system class to be considered.

Definition 1.5 (System class) For $m, r \in \mathbb{N}$ we say that system (1) belongs to the system class $\mathscr{N}^{m, r}$, written $(d, f, \mathbf{T}) \in \mathscr{N}^{m, r}$, if, for some $p, q \in \mathbb{N}$ and $h \geq 0$ the following hold: $d \in L^{\infty}\left(\mathbb{R}_{\geq 0}, \mathbb{R}^{p}\right), f \in C\left(\mathbb{R}^{p} \times \mathbb{R}^{q} \times \mathbb{R}^{m}, \mathbb{R}^{m}\right)$ has the high-gain property, and the operator $\mathbf{T}$ is of class $\mathbb{T}_{h}^{r m, q}$.

We emphasize that the system class $\mathscr{N}^{m, r}$ is parameterized only by two integers, namely, $m$ (which denotes the common dimension of the input and output spaces) and $r$ (which is related to the concept of relative degree). In particular, the class $\mathscr{N}^{m, r}$ encompasses systems with arbitrary state space dimension, including systems with infinite-dimensional internal dynamics, see e.g. [11]: we will elaborate further on this in Sect. 4. 


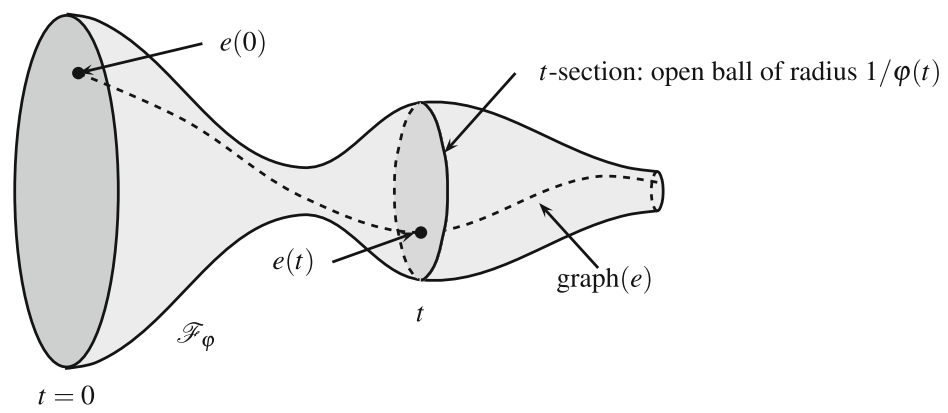

Fig. 1 Performance funnel $\mathscr{F}_{\varphi}$

\subsection{Control objectives}

The control problem to be addressed is to determine an output derivative feedback strategy which ensures that, for every system of class (1) and any reference signal $y_{\text {ref }} \in W^{r, \infty}\left(\mathbb{R}_{\geq 0}, \mathbb{R}^{m}\right)$, the output $y$ approaches the reference $y_{\text {ref }}$ with prescribed transient behaviour and asymptotic accuracy. This objective is reflected in the adoption of a so-called "performance funnel", defined by

$$
\mathscr{F}_{\varphi}:=\left\{(t, e) \in \mathbb{R}_{\geq 0} \times \mathbb{R}^{m} \mid \varphi(t)\|e\|<1\right\},
$$

in which the error function $t \mapsto e(t):=y(t)-y_{\text {ref }}(t)$ is required to evolve; see Fig. 1.

The funnel is shaped - through the choice of its boundary (determined by the reciprocal of $\varphi$ ) - in accordance with the specified transient behaviour and asymptotic accuracy; $\varphi$ is assumed to belong to the set

$$
\Phi:=\left\{\begin{array}{l|l}
\varphi \in \mathrm{AC}_{\text {loc }}\left(\mathbb{R}_{\geq 0}, \mathbb{R}_{\geq 0}\right) & \begin{array}{l}
\forall t>0: \varphi(t)>0, \quad \liminf _{t \rightarrow \infty} \varphi(t)>0, \\
\exists c>0:|\dot{\varphi}(t)| \leq c(1+\varphi(t)) \text { for a.a. } t \geq 0
\end{array}
\end{array},,\right.
$$

where $\mathrm{AC}_{\text {loc }}\left(\mathbb{R}_{\geq 0}, \mathbb{R}_{\geq 0}\right)$ denotes the set of locally absolutely continuous functions $f: \mathbb{R}_{\geq 0} \rightarrow \mathbb{R}_{\geq 0}$. Note that, for $t>0$, the funnel $t$-section $\mathscr{F}_{\varphi} \cap\left(\{t\} \times \mathbb{R}^{m}\right)$ is the open ball in $\mathbb{R}^{m}$ of radius $1 / \varphi(t)$.

While it is often convenient to adopt a monotonically shrinking funnel (through the choice of a monotonically increasing function $\varphi$ ), it might be advantageous to widen the funnel over some later time intervals to accommodate, for instance, periodic disturbances or strongly varying reference signals.

\subsection{Funnel control structure}

We outline the design of funnel control for any system (1) of class $\mathscr{N}^{m, r}$.

\section{Information available for feedback}

Throughout, it is assumed that the instantaneous value of the output $y(t)$ and its first $r-1$ derivatives $\dot{y}(t), \ldots, y^{(r-1)}(t)$ are available for feedback. Admissible reference 
signals are functions $y_{\text {ref }} \in W^{r, \infty}\left(\mathbb{R}_{\geq 0}, \mathbb{R}^{m}\right)$. The instantaneous reference value $y_{\text {ref }}(t)$ is assumed to be accessible to the controller and, if $r \geq 2$, then, for some $\hat{r} \in\{1, \ldots, r\}$, the derivatives $\dot{y}_{\text {ref }}(t), \ldots, y_{\text {ref }}^{(\hat{r}-1)}(t)$ are also accessible for feedback. In summary, for some $\hat{r} \in\{1, \ldots, r\}$, the following instantaneous vector is available for feedback purposes:

$$
\mathbf{e}(t)=\left(e^{(0)}(t), \ldots, e^{(\hat{r}-1)}, y^{(\hat{r})}(t), \ldots, y^{(r-1)}(t)\right) \in \mathbb{R}^{r m}, \quad e(t):=y(t)-y_{\mathrm{ref}}(t),
$$

with the notational convention that $e^{(0)} \equiv e$.

Feedback strategy

Preliminary ingredients in the feedback construction, called funnel control design parameters, are:

$$
\left.\begin{array}{l}
\varphi \in \Phi, \text { bounded if } \hat{r}<r, \\
N \in C\left(\mathbb{R}_{\geq 0}, \mathbb{R}\right), \text { a surjection, } \\
\alpha \in C^{1}([0,1),[1, \infty)), \text { a bijection. }
\end{array}\right\}
$$

These functions are open to choice. For notational convenience, we define

$$
\gamma: \mathscr{B} \rightarrow \mathbb{R}^{m}, w \mapsto \alpha\left(\|w\|^{2}\right) w, \quad \text { where } \mathscr{B}:=\left\{w \in \mathbb{R}^{m} \mid\|w\|<1\right\}
$$

Next, we introduce continuous maps $\rho_{k}: \mathscr{D}_{k} \rightarrow \mathscr{B}, k=1, \ldots, r$, recursively as follows:

$$
\left.\begin{array}{l}
\mathscr{D}_{1}:=\mathscr{B}, \quad \rho_{1}: \mathscr{D}_{1} \rightarrow \mathscr{B}, \eta_{1} \mapsto \eta_{1}, \\
\left.\mathscr{D}_{k}:=\left\{\begin{array}{l|l}
\left(\eta_{1}, \ldots, \eta_{k}\right) \in \mathbb{R}^{k m} \mid \begin{array}{l}
\left(\eta_{1}, \ldots, \eta_{k-1}\right) \in \mathscr{D}_{k-1}, \\
\eta_{k}+\gamma\left(\rho_{k-1}\left(\eta_{1}, \ldots, \eta_{k-1}\right)\right) \in \mathscr{B}
\end{array}
\end{array}\right\},\right\} \\
\rho_{k}: \mathscr{D}_{k} \rightarrow \mathscr{B}, \quad\left(\eta_{1}, \ldots, \eta_{k}\right) \mapsto \eta_{k}+\gamma\left(\rho_{k-1}\left(\eta_{1}, \ldots, \eta_{k-1}\right)\right) .
\end{array}\right\}
$$

Note that each of the sets $\mathscr{D}_{k}$ is non-empty and open. With reference to Fig. 2, and with e and $\rho_{r}$ defined by (5) and (8), the funnel controller is given by

$$
u(t)=(N \circ \alpha)\left(\|w(t)\|^{2}\right) w(t), \quad w(t):=\rho_{r}(\varphi(t) \mathbf{e}(t))
$$

Note the striking simplicity of the control (9): proportional feedback of the information vector $w(t)$, with scalar gain. Further comments on its distinctive features are expounded in Example 1.6 and Remarks 1.7 and 1.8.

Example 1.6 Choosing the design parameter triple

$$
\varphi \in \Phi, \quad N: s \mapsto s \sin (s), \quad \alpha: s \mapsto 1 /(1-s),
$$

(with $\varphi$ bounded if $\hat{r}<r$ ), the feedback becomes

$$
u(t)=\left(1-\|w(t)\|^{2}\right)^{-1} \sin \left(\left(1-\|w(t)\|^{2}\right)^{-1}\right) \cdot w(t)
$$




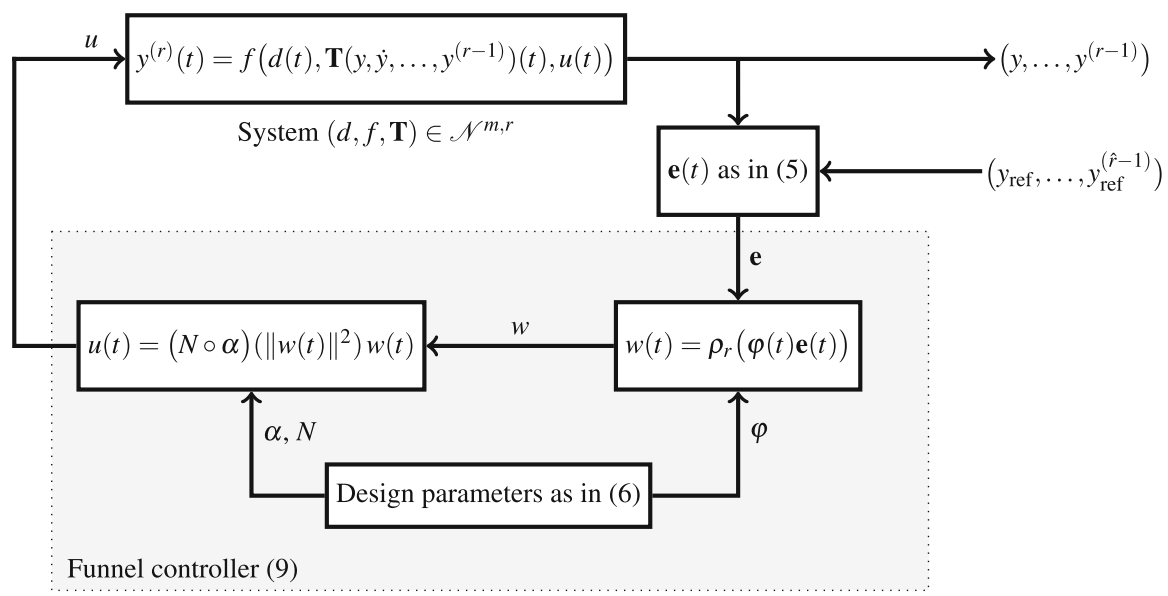

Fig. 2 Construction of the funnel controller (9) depending on its design parameters

where the signal $w(t)$ is, for example,

$$
w(t)= \begin{cases}\varphi(t) e(t), & \text { if } r=1=\hat{r}, \\ \varphi(t) \dot{y}(t)+\gamma(\varphi(t) e(t)), & \text { if } r=2, \hat{r}=1, \\ \varphi(t) \dot{e}(t)+\gamma(\varphi(t) e(t)), & \text { if } r=2, \hat{r}=2, \\ \varphi(t) \ddot{y}(t)+\gamma(\varphi(t) \dot{y}(t)+\gamma(\varphi(t) e(t))), & \text { if } r=3, \hat{r}=1,\end{cases}
$$

with $\gamma$ given by (7).

\section{Remark 1.7}

(a) The intermediate signal $w(t)$ in (9) is a feedback-via the function $\gamma$-of the available information, given by (5), "weighted" by $\varphi(t)$.

(b) We point out that the complexity of the controller is much lower than in previous approaches such as [8], where successive derivatives of auxiliary error variables ${ }^{1}$ need to be calculated before implementation. This complicates the feedback structure for larger values of the parameter $r$. In (9) all required signals are explicitly given by the recursion in (8) and can be implemented directly.

(c) The parameter $\hat{r} \in\{1, \ldots, r\}$ specifies the number of derivatives of $y_{\text {ref }}$ available for feedback. With increasing $\hat{r}$, more information becomes accessible and so, not unreasonably, it might be expected that, loosely speaking, controller performance improves: this expectation is borne out by numerical simulations in Sect. 3 .

(d) Note that, if $\hat{r}=r$, then polynomial or exponentially increasing funnel functions $\varphi$ are admissible. For example, the choices $\varphi: t \mapsto a t^{\ell}$ or $\varphi: t \mapsto e^{a t}-1, a>0$, $\ell \in \mathbb{N}$, ensure polynomial/exponential decay (to zero) of the tracking error $t \mapsto$ $e(t)=y(t)-y_{\text {ref }}(t)$. If $\hat{r}<r$, then boundedness of $\varphi$ is required. As an exemplar in this case, the choice $\varphi: t \mapsto \min \left\{e^{a t}-1, b\right\}, a, b>0$, ensures that the tracking

\footnotetext{
1 The auxiliary error variables are given by $e_{i}(t)$ in equation $(5)$ of [8] for $i=0, \ldots, r-1$.
} 
error approaches the ball of (arbitrarily small) radius $b^{-1}$ exponentially fast and resides in that ball for all $t \geq a^{-1} \ln (1+b)$.

(e) Funnel control presents an anomaly: its performance might seem to contradict the internal model principle which asserts that "a regulator is structurally stable only if the controller [...] incorporates [...] a suitably reduplicated model of the dynamic structure of the exogenous signals which the regulator is required to process" [61, p. 210]. Diverse sources echo this principle-one such source is noted in [26]: a young Mark Twain, when apprenticed to a Mississippi river pilot, recorded the latter's advice on navigating the river in the words "you can always steer by the shape that's in your head, and never mind the one that's before your eyes" [58, Ch. VIII]. But the funnel controller has no "shape" in its "head", it operates only on what is before its eyes. It does not incorporate "a suitably reduplicated model [...] of the exogenous signals". How is this anomaly to be resolved? The internal model principle applies in the context of exact asymptotic tracking of reference signals. In the case of a bounded funnel function $\varphi$, only approximate tracking, with nonzero prescribed asymptotic accuracy, is assured in which case the anomaly is spurious.

(f) But what of the case of an unbounded funnel function $\varphi$, which is permissible whenever $\hat{r}=r$ ? In this case, exact asymptotic tracking is achieved. ( See also the paragraph 'Practical and exact asymptotic tracking' in Sect. 1.1.) Returning to the control-theoretic origins of the internal model principle, summarized in $[61$, p. 210] as "every good regulator must incorporate a model of the outside world", we regard the term "good regulator" as most pertinent. A fundamental ingredient of the funnel controller is the quantity $\varphi(t) \mathbf{e}(t)$ which, in the case of unbounded $\varphi$, inevitably leads to an ill-conditioned computation of the product of "infinitely large" and "infinitesimally small" terms. Such a controller cannot be deemed "good". While of theoretical interest, the case of unbounded $\varphi$ is of limited practical utility.

Remark 1.8 We comment on the function $N \in C\left(\mathbb{R}_{\geq 0}, \mathbb{R}\right)$ in (6).

(a) Note that $N$ is a surjection if, and only if,

$$
\limsup _{s \rightarrow \infty} N(s)=+\infty \text { and } \liminf _{s \rightarrow \infty} N(s)=-\infty
$$

These two conditions are a generalization of the so-called Nussbaum properties (to be discussed further in Sect. 2.2). Reiterating Remark 1.4, the high-gain property implies that, for every pair $\left(K_{p}, K_{q}\right)$ of compact sets, at least one of the conditions in (3) must hold. In the absence of any further a priori knowledge pertaining to these two possibilities, the role of the function $N$ is to provide the controller with a "probing" capability which implicitly accommodates each possibility.

(b) If it is known a priori that $f$ has the negative-definite high-gain property, then $N$ may be replaced by any continuous surjection $[0, \infty) \rightarrow[0, \infty)$, the simplest example being the identity map $s \mapsto s$ in which case the feedback in (9) takes the form $u(t)=\alpha\left(\|w(t)\|^{2}\right) w(t)$.

Similarly, if it is known a priori that $f$ has the positive-definite high-gain property, 


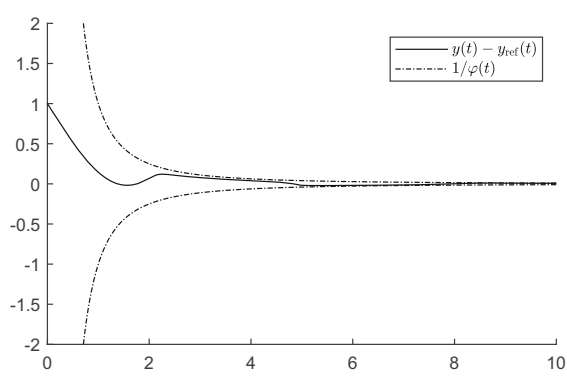

a Funnel and tracking error for $\sigma=-1$

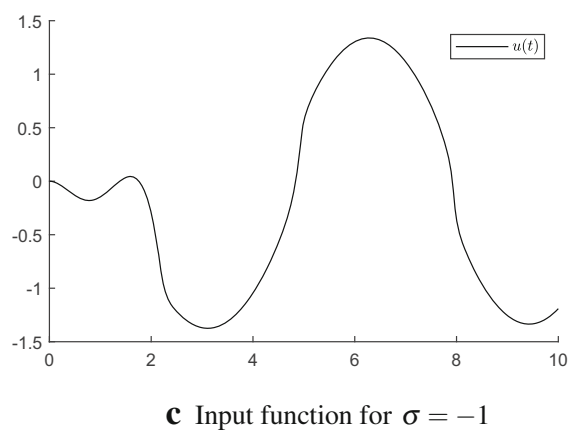

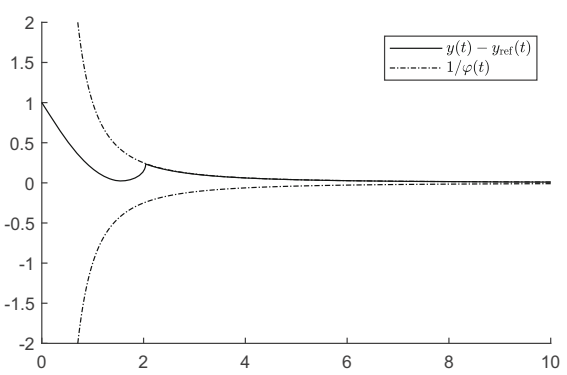

b Funnel and tracking error for $\sigma=+1$

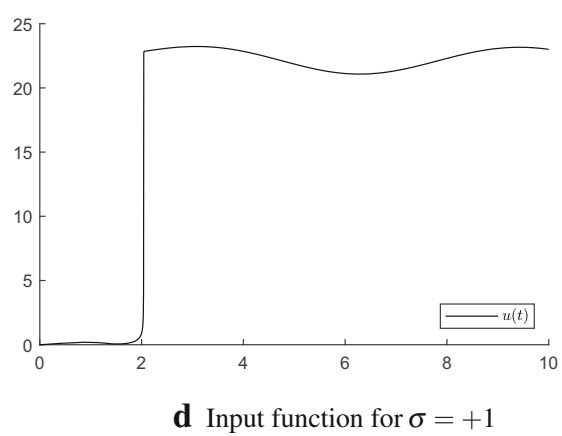

Fig. 3 Simulation of the example from Remark 1.4(a) under control (9) with $N: s \mapsto \sigma s$

then $N$ may be replaced by any continuous surjection $[0, \infty) \rightarrow(-\infty, 0]$, the simplest example being the map $s \mapsto-s$ in which case the feedback takes the form $u(t)=-\alpha\left(\|w(t)\|^{2}\right) w(t)$.

(c) As the example in Remark 1.4(c) shows, it is also possible that (i) and (ii) in (3) hold simultaneously, in which case both of the above simplified feedback laws are feasible. To illustrate this, consider the scalar system

$$
\dot{x}(t)=u(t) \sin (\ln (1+|u(t)|)), \quad x(0)=1,
$$

under control (9) with $\alpha: s \mapsto 1 /(1-s)$ and $N$ replaced by $N: s \mapsto \sigma s$, where $\sigma \in\{-1,1\}$, that is

$$
u(t)=\frac{\sigma w(t)}{1-w(t)^{2}}, \quad w(t)=\varphi(t)\left(y(t)-y_{\mathrm{ref}}(t)\right) .
$$

We choose $\varphi(t)=t^{2}, y_{\text {ref }}(t)=\sin t$ for $t \geq 0$ and perform the simulation ${ }^{2}$ over the time interval $[0,10]$. The results are shown in Fig. 3, where the tracking error and input function for $\sigma=-1$ are depicted in Fig. $3 \mathrm{a}$ and c, and for $\sigma=1$ in Fig. 3b

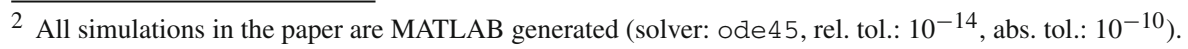


and d, resp. In the latter case, the input exhibits a sharp increase when the tracking error approaches the funnel boundary, and it stays within the interval [20, 25] thereafter, while for $\sigma=-1$ the input stays within the interval $[-1.5,1.5]$. This suggests that the system structure allows the input to "probe" for an appropriate interval of control values, independent of the sign of $\sigma$.

\subsection{Funnel control: main result}

If the funnel controller (9) is applied to a system (1), then the first issue is to prove the existence of solutions of the closed-loop initial-value problem and to establish the efficacy of the control. We stress that the proof is quite delicate-even in the case of linear systems of the form (13). The reason is that the function $\alpha$ used in the feedback (9) introduces a potential singularity on the right hand side of the closed-loop differential equation.

By a solution of $(1),(9)$ on $[-h, \omega)$ we mean a function $y \in C^{r-1}\left([-h, \omega), \mathbb{R}^{m}\right)$, $\omega \in(0, \infty]$, with $\left.y\right|_{[-h, 0]}=y^{0}$ such that $\left.y^{(r-1)}\right|_{[0, \omega)}$ is locally absolutely continuous and satisfies the differential equation in (1) with $u$ defined in (9) for almost all $t \in$ $[0, \omega) ; y$ is said to be maximal, if it has no right extension that is also a solution.

We are now in the position to present the main result for systems belonging to the system class $\mathscr{N}^{m, r}$.

Theorem 1.9 Consider system (1) with $(d, f, \mathbf{T}) \in \mathscr{N}^{m, r}, m, r \in \mathbb{N}$, and initial data $y^{0} \in C^{r-1}\left([-h, 0], \mathbb{R}^{m}\right)$. Let $y_{\mathrm{ref}} \in W^{r, \infty}\left(\mathbb{R}_{\geq 0}, \mathbb{R}^{m}\right)$, with associated parameter $\hat{r} \in\{1, \ldots, r\}$, be arbitrary. Choose the triple $(\alpha, N, \varphi)$ of funnel control design parameters as in (6). Assume that the instantaneous vector $\mathbf{e}(t)$, given by (5), is available for feedback and the following holds:

$$
\varphi(0) \mathbf{e}(0) \in \mathscr{D}_{r},
$$

(trivially satisfied if $\varphi(0)=0$ ).

Then, the funnel control (9) applied to (1) yields an initial-value problem which has a solution, every solution can be maximally extended and every maximal solution $y:[-h, \omega) \rightarrow \mathbb{R}^{m}$ has the properties:

(i) $\omega=\infty$ (global existence);

(ii) $u \in L^{\infty}\left(\mathbb{R}_{\geq 0}, \mathbb{R}^{m}\right), y \in W^{r, \infty}\left([-h, \infty), \mathbb{R}^{m}\right)$;

(iii) the tracking errore $: \mathbb{R}_{\geq 0} \rightarrow \mathbb{R}^{m}$ as in (5) evolves in the funnel $\mathscr{F}_{\varphi}$ and is uniformly bounded away from the funnel boundary

$$
\partial \mathscr{F}_{\varphi}=\left\{(t, \zeta) \in \mathbb{R}_{\geq 0} \times \mathbb{R}^{m} \mid \varphi(t)\|\zeta\|=1\right\}
$$

in the sense that there exists $\varepsilon \in(0,1)$ such that $\varphi(t)\|e(t)\| \leq \varepsilon$ for all $t \geq 0$.

(iv) If $\hat{r}=r$ and $\varphi$ is unbounded, then $e^{(k)}(t) \rightarrow 0$ as $t \rightarrow \infty, k=0, \ldots, r-1$.

(v) If the system is known to satisfy the negative-definite (respectively, positivedefinite) high-gain property (see Remark 1.4(a)), then the feedback (9) may be simplified by substituting the identity map $s \mapsto s$ (respectively, the map $s \mapsto-s$ ) for $N$ and Assertions (i)-(iv) remain valid. 
The proof is relegated to "Appendix A".

When interpreted in specific cases, the initial condition constraint (11) becomes more transparent. For example, in the relative-degree-one case $r=1=\hat{r}$, it is simply the requirement that $\varphi(0)\|e(0)\|<1$, where $e(0)=y^{0}(0)-y_{\text {ref }}(0)$ and, in the case $r=2$, it is equivalent to the same requirement augmented by

$$
\|\varphi(0) z+\gamma(\varphi(0) e(0))\|<1, \quad \text { with } z= \begin{cases}\dot{y}^{0}(0)-\dot{y}_{\mathrm{ref}}(0), & \text { if } \hat{r}=2 \\ \dot{y}^{0}(0), & \text { if } \hat{r}=1\end{cases}
$$

In some specific circumstances, computation of a priori bounds on the evolution of the tracking error $e$ and (some of) its derivatives is possible. We highlight one such circumstance. Assume that $\hat{r} \geq 2$ and $\varphi \in \Phi$ is such that $\varphi(0)>0$. Define

$$
\mu_{0}:=\operatorname{ess} \sup _{t \geq 0}(|\dot{\varphi}(t)| / \varphi(t))
$$

Let $\alpha^{\dagger} \in C^{1}\left(\mathbb{R}_{\geq 0},[0,1)\right)$ denote the inverse of the continuously differentiable bijection $[0,1) \rightarrow \mathbb{R}_{\geq 0}, s \mapsto s \alpha(s)$ and, for notational convenience, introduce the continuous function

$$
\tilde{\alpha}:[0,1) \rightarrow \mathbb{R}_{\geq 0}, s \mapsto 2 s \alpha^{\prime}(s)+\alpha(s) .
$$

Define $\left(\mu_{k}, e_{k}^{0}, c_{k}\right), k=1, \ldots, \hat{r}-1$, recursively as follows:

$$
\begin{aligned}
e_{1}^{0} & :=\varphi(0) e(0), \quad c_{1}:=\max \left\{\left\|e_{1}^{0}\right\|^{2}, \alpha^{\dagger}\left(1+\mu_{0}\right)\right\}^{1 / 2}<1, \quad \mu_{1}:=1+\mu_{0} c_{1}, \\
\mu_{k} & :=1+\mu_{0}\left(1+c_{k-1} \alpha\left(c_{k-1}^{2}\right)\right)+\tilde{\alpha}\left(c_{k-1}^{2}\right)\left(\mu_{k-1}+c_{k-1} \alpha\left(c_{k-1}^{2}\right)\right), \\
e_{k}^{0} & :=\varphi(0) e^{(k-1)}(0)+\alpha\left(\left\|e_{k-1}^{0}\right\|^{2}\right) e_{k-1}^{0}, \\
c_{k} & :=\max \left\{\left\|e_{k}^{0}\right\|^{2}, \alpha^{\dagger}\left(\mu_{k}\right)\right\}^{1 / 2}<1 .
\end{aligned}
$$

We emphasize that the constants $c_{k}$ are determined by the design parameters $\varphi$ and $\alpha$, together with the known initial data: $y(0), \ldots, y^{(\hat{r}-1)}(0)$ and $y_{\mathrm{ref}}(0), \ldots, y_{\text {ref }}^{(\hat{r}-1)}(0)$.

Corollary 1.10 Let all hypotheses of Theorem 1.9 hold. Assume, in addition, that

$$
\hat{r} \geq 2, \quad \varphi(0)>0 \quad \text { and } \alpha^{\prime} \text { is monotonically non-decreasing. }
$$

Then, for every maximal solution $y:[-h, \infty) \rightarrow \mathbb{R}^{m}$ of the feedback system (1) and (9), the tracking error $e=y-y_{\text {ref }}$ and its first $\hat{r}-2$ derivatives satisfy, for all $k=1, \ldots, \hat{r}-2$ and all $t \geq 0$,

$$
\|e(t)\| \leq \varphi(t)^{-1} c_{1}, \quad\left\|e^{(k)}(t)\right\| \leq \varphi(t)^{-1}\left(c_{k+1}+c_{k} \alpha\left(c_{k}^{2}\right)\right) .
$$

where the constants $c_{k}$ are given by (12). 
The proof is relegated to "Appendix A".

Note that these findings are much simpler than the complicated bounds derived in [8, Prop. 3.2].

Example 1.11 Assume $\hat{r}=3, \varphi: t \mapsto a+b t, a, b>0$, and $\alpha: s \mapsto 1 /(1-s)$. In this case, we have $\mu_{0}=b / a$ and $\alpha^{\dagger}: s \mapsto s /(1+s)$. Therefore, for all $t \geq 0$,

$$
e_{1}^{0}=a e(0), \quad c_{1}=\max \left\{\left\|e_{1}^{0}\right\|^{2},\left(1+\mu_{0}\right) /\left(2+\mu_{0}\right)\right\}^{1 / 2} \text { and }\|e(t)\| \leq \frac{c_{1}}{a+b t} .
$$

Furthermore, $\mu_{1}=1+\mu_{0} c_{1}$,

$$
\begin{aligned}
& \tilde{\alpha}: s \mapsto(1+s) /(1-s)^{2}, \quad \mu_{2}=1+\mu_{0}\left(1+c_{1} \alpha\left(c_{1}^{2}\right)\right)+\tilde{\alpha}\left(c_{1}^{2}\right)\left(\mu_{1}+c_{1} \alpha\left(c_{1}^{2}\right)\right), \\
& e_{2}^{0}=a \dot{e}(0)+\left(1-\left\|e_{1}^{0}\right\|^{2}\right)^{-1} e_{1}^{0}, \quad c_{2}=\max \left\{\left\|e_{2}^{0}\right\|^{2}, \mu_{2} /\left(1+\mu_{2}\right)\right\}^{1 / 2} \\
& \text { and } \forall t \geq 0:\|\dot{e}(t)\| \leq \frac{c_{2}+c_{1} /\left(1-c_{1}^{2}\right)}{a+b t} .
\end{aligned}
$$

\section{Examples}

In this section, we show that the class $\mathscr{N}^{m, r}$ encompasses the prototype of linear multi-input multi-output systems with strict relative degree $r \in \mathbb{N}$ and asymptotically stable zero dynamics, see Sect. 2.1. Furthermore, the issues of control directions are discussed in Sect. 2.2 and input nonlinearities in Sect. 2.3; a special case of the latter is a so called dead-zone input which is discussed in Sect. 2.4.

\subsection{The prototypical linear system class}

As a concrete example we consider linear, finite-dimensional systems of the form

$$
\left.\begin{array}{l}
\dot{x}(t)=A x(t)+B u(t), \quad x(0)=x^{0} \in \mathbb{R}^{n}, \\
y(t)=C x(t)
\end{array}\right\}
$$

where $(A, B, C) \in \mathbb{R}^{n \times n} \times \mathbb{R}^{n \times m} \times \mathbb{R}^{m \times n}, m \leq n$, and discuss its relationships to Properties (TP1)-(TP3) and the high-gain property.

\subsubsection{Strict relative degree}

We show that system (13) can be equivalently written in the form (1), if system (13) has (strict) relative degree $r \in \mathbb{N}$, that is

$$
C A^{k} B=0, k=0, \ldots, r-2 \text { and } \Gamma:=C A^{r-1} B \text { is invertible. }
$$


It is shown in [32] that under this assumption there exists a state space transformation

$$
z=\left(\begin{array}{c}
\xi \\
\eta
\end{array}\right)=U x, \quad \xi=\left(\begin{array}{c}
\xi_{1} \\
\vdots \\
\xi_{r}
\end{array}\right), \quad U \in \mathbb{R}^{n \times n} \text { invertible }
$$

which transforms (13) into Byrnes-Isidori form

$$
\dot{z}(t)=\widetilde{A} z(t)+\widetilde{B} u(t), \quad y(t)=\widetilde{C} z(t),
$$

where

$$
(\widetilde{A}, \widetilde{B}, \widetilde{C})=\left(U A U^{-1}, U B, C U^{-1}\right)
$$

with

$$
\begin{aligned}
\widetilde{A} & =\left[\begin{array}{cccccc}
0 & I_{m} & 0 & \ldots & 0 & 0 \\
0 & 0 & I_{m} & & & 0 \\
\vdots & & \ddots & \ddots & & \vdots \\
0 & 0 & \ldots & 0 & I_{m} & 0 \\
R_{1} & R_{2} & \ldots & R_{r-1} & R_{r} & S \\
P & 0 & \ldots & 0 & 0 & Q
\end{array}\right], \quad \widetilde{B}=\left[\begin{array}{c}
0_{m \times m} \\
\vdots \\
0_{m \times m} \\
\Gamma \\
0_{(n-r m) \times m}
\end{array}\right], \\
\widetilde{C} & =\left[\begin{array}{lllll}
I_{m}, & 0_{m \times m}, & \ldots & 0_{m \times m}, & 0_{m \times(n-r m)}
\end{array}\right] .
\end{aligned}
$$

In the new coordinates, the system representation of (13) becomes

$$
\left.\begin{array}{l}
\dot{\xi}_{k}(t)=\xi_{k+1}(t), \quad k=1, \ldots, r-1, \\
\dot{\xi}_{r}(t)=\sum_{k=1}^{r} R_{k} \xi_{k}(t)+S \eta(t)+\Gamma u(t), \\
\dot{\eta}(t)=P \xi_{1}(t)+Q \eta(t)
\end{array}\right\} \quad \text { with output } y(t)=\xi_{1}(t)
$$

With the third equation in (15), the so-called internal dynamics, we may associate a linear operator

$$
L: y(\cdot) \mapsto\left(t \mapsto \int_{0}^{t} \mathrm{e}^{Q(t-\tau)} P y(\tau) \mathrm{d} \tau\right)
$$

With initial data $\eta(0)=\eta^{0}=\left[0, I_{n-r m}\right] U x^{0}$ and $d(\cdot):=\mathrm{e}^{Q \cdot} \eta^{0}$, we find that

$$
\eta(t)=d(t)+L(y)(t)
$$

Introducing the (linear) operator

$$
\begin{aligned}
& \mathbf{T}: C\left(\mathbb{R}_{\geq 0}, \mathbb{R}^{r m}\right) \rightarrow L_{\mathrm{loc}}^{\infty}\left(\mathbb{R}_{\geq 0}, \mathbb{R}^{m}\right), \\
& \zeta=\left(\zeta_{1}, \ldots, \zeta_{r}\right) \mapsto\left(t \mapsto \sum_{k=1}^{r} R_{k} \zeta_{k}(t)+S L\left(\zeta_{1}\right)(t)\right),
\end{aligned}
$$


it follows from (15) that (13) is equivalent to the functional differential system

$$
\left.\begin{array}{l}
y^{(r)}(t)=S d(t)+\mathbf{T}\left(y, \ldots, y^{(r-1)}\right)(t)+\Gamma u(t) \\
y(0)=C x^{0}, \ldots, y^{(r-1)}(0)=C A^{r-1} x^{0} .
\end{array}\right\}
$$

It is easy to see that the operator $\mathbf{T}$ satisfies properties (TP1) and (TP2) from Definition 1.1. The following section is devoted to (TP3).

\subsubsection{Minimum phase}

Suppose that system (13) has strict relative degree $r \in \mathbb{N}$. Then the BIBO property (TP3) of the operator $\mathbf{T}$ in (18) is closely related to system (13) having asymptotically stable zero dynamics, i.e.

$$
\forall \lambda \in \mathbb{C}_{\geq 0}: \operatorname{det}\left[\begin{array}{cc}
\lambda I-A & B \\
C & 0
\end{array}\right] \neq 0 .
$$

This concept (also closely related to the minimum phase property in the literature, cf. [34]) is extensively studied since its relevance has been revealed in classical works such as $[17,40]$. To be precise, assume that the transfer function $C(s I-A)^{-1} B \in$ $\mathbb{R}(s)^{m \times m}$ of $(A, B, C)$ is invertible over $\mathbb{R}(s)$, then we have the following:
$(A, B, C)$ satisfies (19)

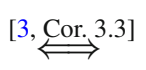
$(A, B, C)$ stabilizable and detectable, $C(s I-A)^{-1} B$ has no zeros in $\mathbb{C}_{\geq 0}$
介 $[3$, Cor. 2.8]
( $A, B, C)$ stab. and det.,
[57, $\underset{\text { Thm. }}{\Longleftrightarrow} 3.21]$
$(A, B, C)$ stabilizable and detectable, $S(s I-Q)^{-1} P$ has no poles in $\mathbb{C}_{\geq 0}$

For the last equivalence above we note that by [57, Thm. 3.21] it is straightforward that $S(s I-Q)^{-1} P$ having no poles in $\mathbb{C}_{\geq 0}$ is equivalent to $(Q, P, S)$ being externally stable or, in other words, the operator $L$ from (16) satisfies (TP3). It is easily seen that this is the same as $\mathbf{T}$ satisfying (TP3).

\subsubsection{Sign-definite high-frequency gain matrix}

We show that system (13) satisfies the high-gain property (recall Definition 1.2) if, and only if, the high-frequency gain matrix $\Gamma=C A^{r-1} B$ is sign definite. Otherwise stated, we seek to establish the following equivalence:

(a) (13) has the high-gain property $\Longleftrightarrow$ (b) $\forall v \in \mathbb{R}^{m} \backslash\{0\}: v^{\top} \Gamma v \neq 0$.

(a) $\Longrightarrow$ (b): Assume (a). Let $v^{*} \in(0,1)$ be given and choose $K_{p}=\{0\}, K_{q}=\{0\}$. Write $A_{m}:=\left\{v \in \mathbb{R}^{m} \mid v^{*} \leq\|v\| \leq 1\right\}$. Suppose (b) is false. Then there exists $\hat{v} \in$ 
$A_{m}$ such that $\hat{v}^{\top} \Gamma \hat{v}=0$, thus

$$
\forall s \in \mathbb{R}: \chi(s)=\min _{v \in A_{m}}\left(-s v^{\top} \Gamma v\right) \leq-s \hat{v}^{\top} \Gamma \hat{v}=0
$$

which contradicts (a).

(b) $\Longrightarrow$ (a): Assume (b). Then there exists $\sigma \in\{-1,1\}$ such that $\sigma \Gamma$ is positive definite. Let $G:=(\sigma / 2)\left(\Gamma+\Gamma^{\top}\right)$ denote the symmetric part of $\sigma \Gamma$ and let $\lambda_{*}>0$ be the smallest eigenvalue of $G$. Set $v^{*}=\frac{1}{2}$, choose compact $K_{p} \subset \mathbb{R}^{p}$ and $K_{q} \subset \mathbb{R}^{q}$ and define

$$
c_{1}:=\min \left\{v^{\top}(\delta+z) \mid(\delta, z, v) \in K_{p} \times K_{q} \times A_{m}\right\} .
$$

Then,

$$
\forall s \in \mathbb{R}: \chi(s)-c_{1} \geq \min _{v \in A_{m}}\left(-s v^{\top} \Gamma v\right)=\min _{v \in A_{m}}\left(-s \sigma v^{\top} G v\right)
$$

Let $\left(s_{n}\right)$ be a real sequence with $\sigma s_{n}<0$ for all $n \in \mathbb{N}$ and $\sigma s_{n} \rightarrow-\infty$ as $n \rightarrow \infty$. It follows that

$$
\forall n \in \mathbb{N} \forall v \in A_{m}:-\sigma s_{n} v^{\top} G v \geq-\sigma s_{n} \lambda_{*}\|v\|^{2} \geq-\frac{\sigma s_{n} \lambda_{*}}{4}
$$

and so we have

$$
\forall n \in \mathbb{N}: \chi\left(s_{n}\right) \geq c_{1}-\frac{\sigma s_{n} \lambda_{*}}{4} .
$$

Therefore, $\chi\left(s_{n}\right) \rightarrow \infty$ as $n \rightarrow \infty$ and so (a) holds.

\subsection{Known and unknown control directions}

For linear systems (13) with relative degree $r \in \mathbb{N}$ the notion of "control direction" is captured by the sign of the high-frequency gain matrix $\Gamma=C A^{r-1} B$ as discussed in Sect. 2.1.3. More precisely, if $\sigma \Gamma$ is positive definite for some $\sigma \in\{-1,1\}$, then $\sigma$ is called the control direction. If $\sigma$ is known and the system (13) has asymptotically stable zero dynamics, see (19), then it can be shown that the "classical high-gain adaptive feedback"

$$
u(t)=-\sigma k(t) y(t), \quad \dot{k}(t)=\|y(t)\|^{2},
$$

with $k(0)=k^{0} \geq 0$, applied to (13) yields a closed-loop system, where for any solution $(x, k)$ we have that $x(t) \rightarrow 0$ as $t \rightarrow \infty$ and $k(\cdot)$ is bounded; see [17,40,42].

For the case of unknown control direction $\sigma$, the adaptive stabilization was an obstacle over many years. Morse [42] conjectured the non-existence of a smooth adaptive controller which stabilizes every linear single-input single-output system (13), i.e. $m=1$, under the assumption that $\Gamma \neq 0$. It was shown by Nussbaum in [44] 
that this conjecture is false: One has to incorporate a "sign-sensing function" in the feedback law (20) so that it becomes

$$
u(t)=-N(k(t)) y(t), \quad \dot{k}(t)=\|y(t)\|^{2},
$$

where the smooth function $N: \mathbb{R}_{\geq 0} \rightarrow \mathbb{R}$ satisfies the so-called Nussbaum property

$$
\forall k^{0} \geq 0: \sup _{k>k^{0}} \frac{1}{k-k^{0}} \int_{k^{0}}^{k} N(\kappa) \mathrm{d} \kappa=\infty \quad \text { and } \inf _{k>k^{0}} \frac{1}{k-k^{0}} \int_{k^{0}}^{k} N(\kappa) \mathrm{d} \kappa=-\infty,
$$

see, for example, [18-20,36,62]. Loosely speaking, when incorporated in the control design, "Nussbaum" functions provide a mechanism that can "probe" in both control directions.

The present paper utilizes a larger class of "probing" functions: in particular, the proposed control design permits the adoption of any continuous function $N: \mathbb{R}_{\geq 0} \rightarrow \mathbb{R}$ which is surjective or, equivalently, satisfies (10). Properties (22) imply properties (10), but the reverse implication is false: for example, the function $s \mapsto N(s)=s \sin s$ exhibits properties (10), but fails to exhibit the Nussbaum properties (22).

\subsection{Input nonlinearities}

In addition to accommodating the issue of (unknown) control direction (cf. Sect. 2.2), the generic formulation (1) with associated high-gain property encompasses a wide variety of input nonlinearities. Consideration of a scalar system of the simple form

$$
\dot{y}(t)=f_{1}(y(t))+f_{2}(y(t)) \beta(u(t))
$$

with $f_{1} \in C(\mathbb{R}, \mathbb{R}), f_{2} \in C(\mathbb{R}, \mathbb{R} \backslash\{0\})$ and $\beta \in C(\mathbb{R}, \mathbb{R})$, will serve to illustrate this variety. The assumption that $f_{2}$ is a non-zero-valued continuous function ensures a well-defined control direction (unknown to the controller). Without loss of generality, we may assume that $f_{2} \in C\left(\mathbb{R}, \mathbb{R}_{>0}\right)$; if $f_{2}$ is negative-valued, then, in (23), simply replace $f_{2}$ by $-f_{2}$ and $\beta$ by $-\beta$. We impose the following conditions on $\beta \in C(\mathbb{R}, \mathbb{R})$ :

$$
\beta \text { is surjective, with }|\beta(\tau)| \rightarrow \infty \text { as }|\tau| \rightarrow \infty,
$$

which is equivalent to the requirement that one of the following conditions hold:

$$
\lim _{\tau \rightarrow \pm \infty} \beta(\tau)= \pm \infty \text { or } \lim _{\tau \rightarrow \pm \infty} \beta(\tau)=\mp \infty
$$

We proceed to show that system (23) has the high-gain property. Set $v^{*}=\frac{1}{2}$, let $K_{1} \subset$ $\mathbb{R}$ be compact and define

$$
A_{1}:=\left[-1,-\frac{1}{2}\right] \cup\left[\frac{1}{2}, 1\right], \quad c_{1}:=\min \left\{v f_{1}(z) \mid(z, v) \in K_{1} \times A_{1}\right\} \in \mathbb{R}
$$


Consider the function

$$
\chi: \mathbb{R} \rightarrow \mathbb{R}, \quad s \mapsto \min \left\{v\left(f_{1}(z)+f_{2}(z) \beta(-s v)\right) \mid(z, v) \in K_{1} \times A_{1}\right\} .
$$

Then,

$$
\forall s \in \mathbb{R}: \chi(s) \geq c_{1}+\min \left\{v f_{2}(z) \beta(-s v) \mid(z, v) \in K_{1} \times A_{1}\right\}
$$

Let $M>0$ be arbitrary. To conclude that the high-gain property holds, it suffices to show that there exists $s \in \mathbb{R}$ such that

$$
\forall(z, v) \in K_{1} \times A_{1}: v f_{2}(z) \beta(-s v)>M .
$$

Define

$$
c_{2}:=\min _{z \in K_{1}} f_{2}(z)>0 \text { and } c_{3}:=2 M / c_{2}
$$

By properties of $\beta$, there exist $\sigma \in\{-1,1\}$ and $c_{4}>0$ such that

$$
\forall \tau>c_{4}: \min \{\beta(\sigma \tau),-\beta(-\sigma \tau)\}>c_{3} .
$$

Let $(z, v) \in K_{1} \times A_{1}$ be arbitrary. Fix $s \in \mathbb{R}$ such that $\sigma s<-2 c_{4}$ and so $|s v|>c_{4}$. Then,

$$
v f_{2}(z) \beta(-s v)=\left\{\begin{array}{ll}
|v| f_{2}(z) \beta(\sigma|s v|), & \text { if } v>0 \\
|v| f_{2}(z)(-\beta(-\sigma|s v|)), & \text { if } v<0
\end{array}\right\}>\frac{c_{2} c_{3}}{2}=M
$$

Therefore, the high-gain property holds.

\subsection{Dead-zone input}

An important example of a nonlinearity $\beta=D$ with properties (24) is a so-called dead-zone input of the form

$$
D: \mathbb{R} \rightarrow \mathbb{R}, \quad v \mapsto D(v)= \begin{cases}D_{r}(v), & v \geq b_{r} \\ 0, & b_{l}<v<b_{r} \\ D_{l}(v), & v \leq b_{l}\end{cases}
$$

with unknown deadband parameters $b_{l}<0<b_{r}$ and unknown functions $D_{l}, D_{r} \in$ $C(\mathbb{R}, \mathbb{R})$ which satisfy, for unknown $\sigma \in\{-1,1\}$,

$$
D_{l}\left(b_{l}\right)=D_{r}\left(b_{r}\right)=0 \text { and } \lim _{s \rightarrow \infty} \sigma D_{r}(s)=\infty, \quad \lim _{s \rightarrow-\infty} \sigma D_{l}(s)=-\infty .
$$

Note that the above assumptions allow for a much larger class of functions $D_{l}, D_{r}$ compared to e.g. [43], where assumptions on their derivatives are used. In particular, in the present context, $D_{l}$ and $D_{r}$ need not be differentiable or monotone. 


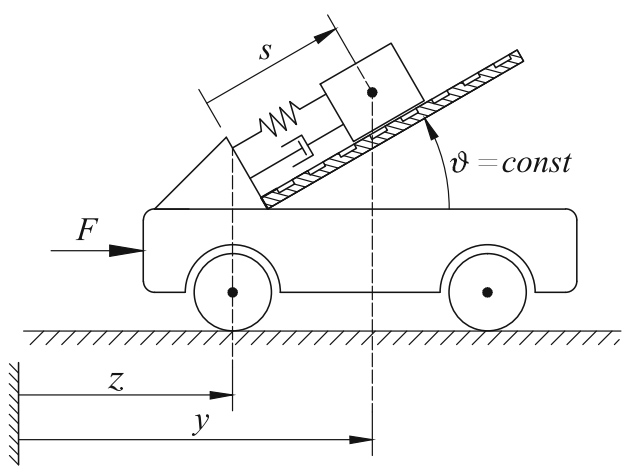

Fig. 4 Mass-on-car system

\section{Simulations}

We compare the controller (9) to the controller presented in [8] and, to this end, consider the simulation examples presented therein.

\subsection{Mass-on-car system}

To illustrate the controller (9), we consider a mass-spring system mounted on a car from [51], see Fig. 4. The mass $m_{2}$ (in $\mathrm{kg}$ ) moves on a ramp inclined by the angle $\vartheta \in$ $\left[0, \frac{\pi}{2}\right.$ ) (in rad) and mounted on a car with mass $m_{1}$ (in $\mathrm{kg}$ ), for which it is possible to control the force with $u=F$ (in $\mathrm{N}$ ) acting on it. The equations of motion for the system are given by

$$
\left[\begin{array}{cc}
m_{1}+m_{2} & m_{2} \cos \vartheta \\
m_{2} \cos \vartheta & m_{2}
\end{array}\right]\left(\begin{array}{l}
\ddot{z}(t) \\
\ddot{s}(t)
\end{array}\right)+\left(\begin{array}{c}
0 \\
k s(t)+d \dot{s}(t)
\end{array}\right)=\left(\begin{array}{c}
u(t) \\
0
\end{array}\right)
$$

where $t$ is current time (in s), $z$ (in $\mathrm{m}$ ) is the horizontal car position and $s$ (in $\mathrm{m}$ ) the relative position of the mass on the ramp. The constants $k>0$ (in $\mathrm{N} / \mathrm{m}$ ), $d>0$ (in $\mathrm{Ns} / \mathrm{m}$ ) are the coefficients of the spring and damper, respectively. The output $y$ (in $\mathrm{m}$ ) of the system is given by the horizontal position of the mass on the ramp,

$$
y(t)=z(t)+s(t) \cos \vartheta .
$$

Writing $\mu:=m_{2}\left(m_{1}+m_{2} \sin ^{2} \vartheta\right), \mu_{1}:=m_{1} / \mu$ and $\mu_{2}:=m_{2} / \mu$, it is readily verified that this system takes the form (13), with

$$
x(t):=\left(\begin{array}{c}
z(t) \\
\dot{z}(t) \\
s(t) \\
\dot{s}(t)
\end{array}\right), \quad A:=\left[\begin{array}{cccc}
0 & 1 & 0 & 0 \\
0 & 0 & \mu_{2} k \cos \vartheta & \mu_{2} d \cos \vartheta \\
0 & 0 & 0 & 1 \\
0 & 0 & -\left(\mu_{1}+\mu_{2}\right) k & -\left(\mu_{1}+\mu_{2}\right) d
\end{array}\right], \quad B:=\left[\begin{array}{c}
0 \\
\mu_{2} \\
0 \\
-\mu_{2} \cos \vartheta
\end{array}\right]
$$


and $C:=\left[\begin{array}{llll}1 & 0 & \cos \vartheta & 0\end{array}\right]$. Observe that

$$
C B=0, \quad C A B=\mu_{2} \sin ^{2} \vartheta, \quad C A^{2} B=d \mu_{1} \mu_{2} \cos ^{2} \vartheta
$$

and so the relative degree $r$ of the system is given by

$$
r= \begin{cases}2, & \text { if } \vartheta \in\left(0, \frac{\pi}{2}\right) \\ 3, & \text { if } \vartheta=0 .\end{cases}
$$

Moreover, $C A^{r-1} B>0$ and so the positive-definite high-gain property holds. Furthermore, a straightforward (if tedious) calculation reveals that the eigenvalues of the matrix $Q \in \mathbb{R}^{(4-r) \times(4-r)}$ in the Byrnes-Isidori form (15) are given by

$$
\lambda:=-k / d \text { in the case } r=3
$$

or, in the case $r=2$, by

$$
\lambda_{ \pm}:=-(\tilde{d} / 2) \pm \sqrt{(\tilde{d} / 2)^{2}-\tilde{k}}, \quad \tilde{d}:=d /\left(m_{2} \sin ^{2} \vartheta\right), \quad \tilde{k}:=k /\left(m_{2} \sin ^{2} \vartheta\right)
$$

Thus, in each case, the zero dynamics are asymptotically stable and so property (TP3) holds for the associated operator $\mathbf{T}$ given by (17). Therefore, the system is of class $\mathscr{N}^{1, r}$ to which the funnel control (9) applies. Invoking Assertion(v) of Theorem 1.9, the function $N$ in (9) may be substituted by the map $s \mapsto-s$.

For the simulation, we choose the parameters $m_{1}=4, m_{2}=1, k=2, d=1$, the initial values $x(0)=s(0)=0, \dot{x}(0)=\dot{s}=0$ and the reference trajectory $y_{\mathrm{ref}}: t \mapsto \cos t$. We emphasize that the function $y_{\mathrm{ref}}(\cdot)$ is not available a priori to the controller: all that is available is the function value at the current time $t$ together with the values of its first $\hat{r}-1$ derivatives, $y_{\text {ref }}^{(i)}(t), i=0, \ldots, \hat{r}-1$. We consider two cases.

Case 1 If $0<\vartheta<\frac{\pi}{2}$, then system (26) has relative degree $r=2$, and the funnel controller (9) with $\hat{r}=r=2$ is

$$
u(t)=-\alpha\left(w(t)^{2}\right) w(t) \text {, with } w(t)=\varphi(t) \dot{e}(t)+\alpha\left(\varphi(t)^{2} e(t)^{2}\right) \varphi(t) e(t),
$$

where $\alpha(s)=1 /(1-s)$ for $s \in[0,1)$. The controller presented in [8] takes the form

$$
u(t)=-\alpha\left(\varphi_{1}(t)^{2} w_{1}(t)^{2}\right) w_{1}(t), \text { with } w_{1}(t)=\dot{e}(t)+\alpha\left(\varphi(t)^{2} e(t)^{2}\right) e(t)
$$

where $\varphi_{1}$ is a second funnel function, chosen appropriately, cf. [8]. Note that $w(t)=\varphi(t) w_{1}(t)$. As simulations show, the performance of the controller (27) can be improved compared to the simulations in [8], by choosing $\varphi_{1}=\varphi$. As in [8], we set $\varphi(t)=\left(5 e^{-2 t}+0.1\right)^{-1}$ for $t \geq 0$.

The performance of the controllers (9) and (27) applied to (26) is depicted in Fig. 5. Figure 5 a shows the tracking errors generated by the two different controllers, while 


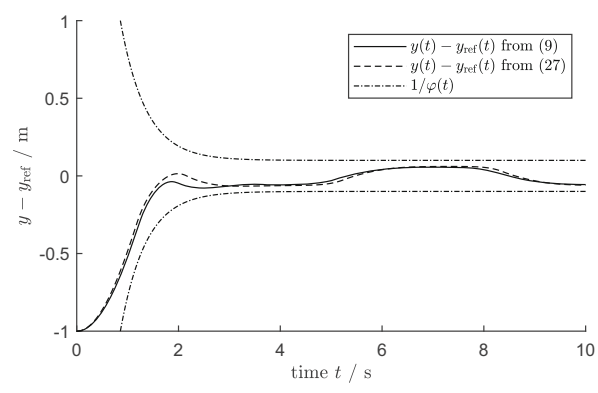

a Funnel and tracking errors

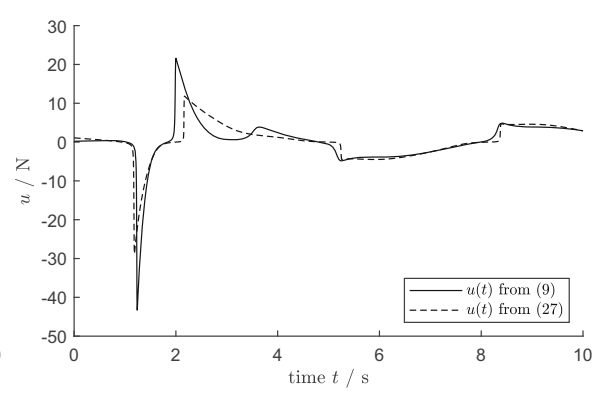

b Input functions

Fig. 5 Simulation, under controllers (9) and (27), of system (26) with $\vartheta=\frac{\pi}{4}$

Fig. 5b shows the respective input functions. Comparable performance is evident, suggesting broadly similar efficacy in cases wherein both controllers are feasible. However, (9) is feasible in certain situations which are outside the scope of (27). For example, (9) is able to achieve asymptotic tracking, to address the issue of an unknown control direction and is applicable when the instantaneous value $\dot{y}_{\text {ref }}(t)$ is not available to the controller: these features form the basis of the example in Sect. 3.3.

Case 2 If $\vartheta=0$ and $d \neq 0$, then system (26) has relative degree $r=3$. Then, the funnel controller (9), with $\hat{r}=r=3$, takes the form

$$
\begin{aligned}
w(t) & =\varphi(t) \ddot{e}(t)+\gamma(\varphi(t) \dot{e}(t)+\gamma(\varphi(t) e(t))), \\
u(t) & =-\gamma(w(t)),
\end{aligned}
$$

where $\gamma(s)=s \alpha\left(s^{2}\right)$ for $s \in(-1,1)$. The controller presented in [8] reads

$$
\begin{aligned}
w_{1}(t)= & \dot{e}(t)+\alpha\left(\varphi(t)^{2} e(t)^{2}\right) e(t) \\
w_{2}(t)= & \dot{w}_{1}(t)+\alpha\left(\varphi_{1}(t)^{2} w_{1}(t)^{2}\right) w_{1}(t) \\
= & \ddot{e}(t)+2 \alpha\left(\varphi(t)^{2} e(t)^{2}\right)^{2}\left(\dot{\varphi}(t) \varphi(t)\|e(t)\|^{2}+\varphi(t)^{2} e(t)^{\top} \dot{e}(t)\right) e(t) \\
& +\alpha\left(\varphi(t)^{2} e(t)^{2}\right) \dot{e}(t)+\alpha\left(\varphi_{1}(t)^{2} w_{1}(t)^{2}\right) w_{1}(t) \\
u(t)= & -\alpha\left(\varphi_{2}(t)^{2} w_{2}(t)^{2}\right) w_{2}(t)
\end{aligned}
$$

where $\varphi_{1}, \varphi_{2}$ are appropriate additional funnel functions, cf. [8]. Here, we choose $\varphi_{1}=\varphi_{2}=\varphi$, with $\varphi(t)=\left(3 e^{-t}+0.1\right)^{-1}$ for $t \geq 0$ and compare the controller (9) with (28).

The simulation (Fig. 6) suggests that the controllers are broadly similar in performance. While controller (9) requires more input action than controller (28), the latter exhibits a significantly higher level of complexity, which makes it more difficult to implement (this issue becomes even more severe for relative degrees higher than three). 


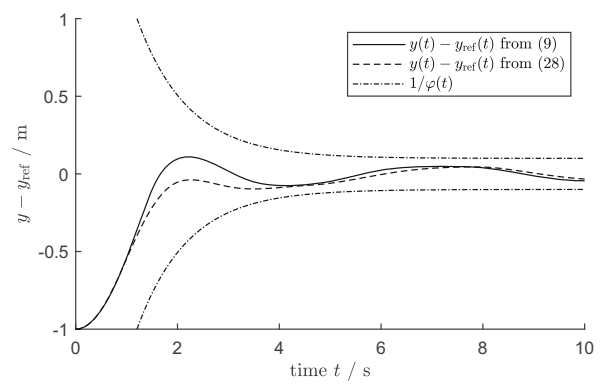

a Funnel and tracking errors

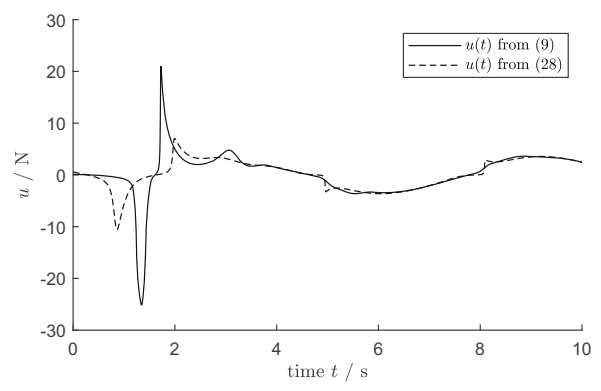

b Input functions

Fig. 6 Simulation, under controllers (9) and (28), of system (26) with $\vartheta=0$

Fig. 7 Planar rigid revolute joint robotic manipulator

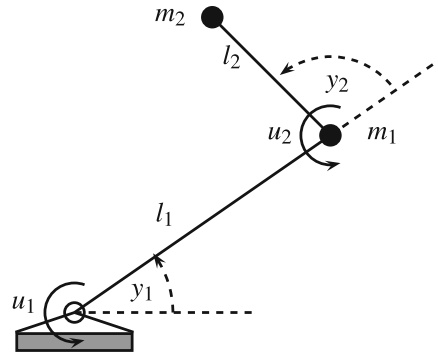

\subsection{Nonlinear MIMO system}

As a nonlinear multi-input, multi-output example, we consider the robotic manipulator from [24, Ch. 13] as depicted in Fig. 7. It is planar, rigid, with revolute joints and has two degrees of freedom.

The two joints are actuated by $u_{1}$ and $u_{2}$ (in Nm). The links are assumed to be massless and have lengths $l_{1}$ and $l_{2}$ (in $\mathrm{m}$ ), resp., with point masses $m_{1}$ and $m_{2}$ (in $\mathrm{kg}$ ) attached to their ends. The two outputs are the joint angles $y_{1}$ and $y_{2}$ (in rad) and the equations of motion are given by (see also [54, p. 259])

$$
M(y(t)) \ddot{y}(t)+C(y(t), \dot{y}(t)) \dot{y}(t)+G(y(t))=u(t)
$$

with initial value $(y(0), \dot{y}(0))=(0,0)$, inertia matrix

$$
M: \mathbb{R}^{2} \rightarrow \mathbb{R}^{2 \times 2},\left(y_{1}, y_{2}\right) \mapsto\left[\begin{array}{cc}
m_{1} l_{1}^{2}+m_{2}\left(l_{1}^{2}+l_{2}^{2}+2 l_{1} l_{2} \cos \left(y_{2}\right)\right) & m_{2}\left(l_{2}^{2}+l_{1} l_{2} \cos \left(y_{2}\right)\right) \\
m_{2}\left(l_{2}^{2}+l_{1} l_{2} \cos \left(y_{2}\right)\right) & m_{2} l_{2}^{2}
\end{array}\right]
$$

centrifugal and Coriolis force matrix

$$
C: \mathbb{R}^{4} \rightarrow \mathbb{R}^{2 \times 2},\left(y_{1}, y_{2}, v_{1}, v_{2}\right) \mapsto\left[\begin{array}{cc}
-2 m_{2} l_{1} l_{2} \sin \left(y_{2}\right) v_{1} & -m_{2} l_{1} l_{2} \sin \left(y_{2}\right) v_{2} \\
-m_{2} l_{1} l_{2} \sin \left(y_{2}\right) v_{1} & 0
\end{array}\right]
$$




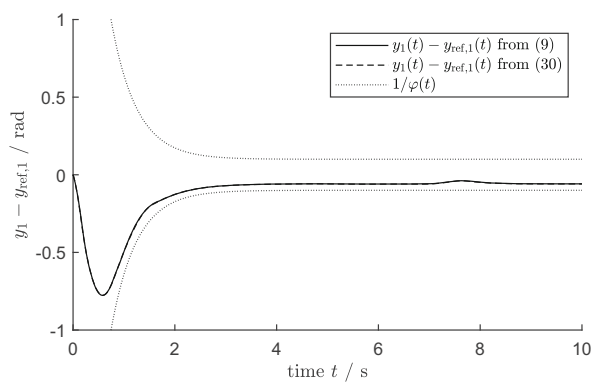

a Funnel and first tracking error components

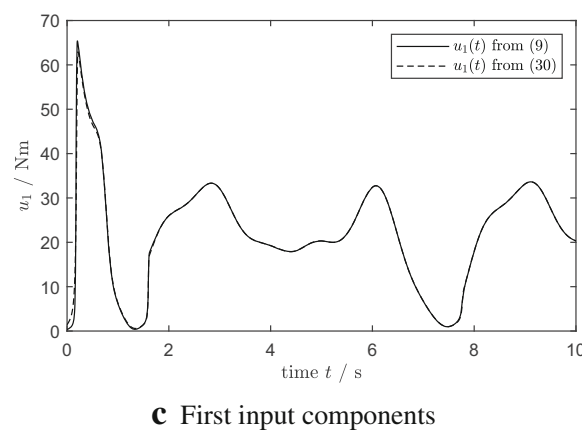

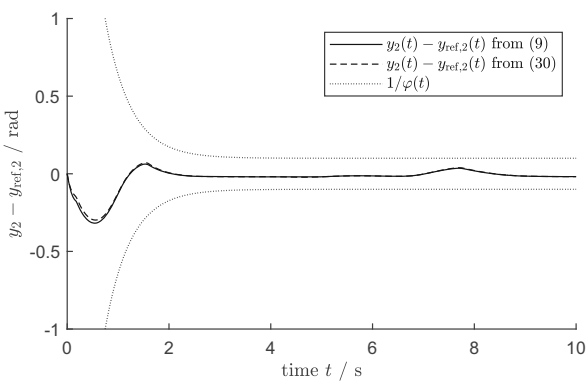

b Funnel and second tracking error components

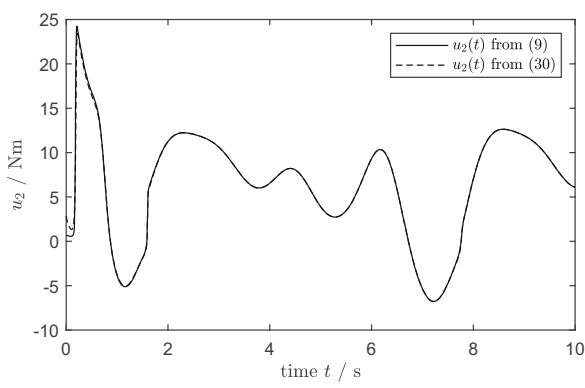

d Second input components

Fig. 8 Simulation of the controllers (9) and (30) applied to (29)

and gravity vector

$$
G: \mathbb{R}^{2} \rightarrow \mathbb{R}^{2},\left(y_{1}, y_{2}\right) \mapsto g\left(\begin{array}{c}
m_{1} l_{1} \cos \left(y_{1}\right)+m_{2}\left(l_{1} \cos \left(y_{1}\right)+l_{2} \cos \left(y_{1}+y_{2}\right)\right) \\
m_{2} l_{2} \cos \left(y_{1}+y_{2}\right)
\end{array}\right)
$$

where $g=9.81 \mathrm{~m} / \mathrm{s}^{2}$ is the acceleration of gravity. Multiplying (29) with $M(y(t))^{-1}$, which is pointwise positive definite, from the left we see that the resulting system is of the form (1) and satisfies the positive-definite high-gain property, hence it belongs to $\mathscr{N}^{2,2}$.

For the simulation, we choose the parameters $m_{1}=m_{2}=1, l_{1}=l_{2}=1$ and the reference signal $y_{\text {ref }}: t \mapsto(\sin t, \sin 2 t)$. We compare the controller (9) to the multivariate version of (27) from [8], that is

$$
u(t)=-\alpha\left(\varphi_{1}(t)^{2}\left\|w_{1}(t)\right\|^{2}\right) w_{1}(t), \quad \text { with } \quad w_{1}(t)=\dot{e}(t)+\alpha\left(\varphi(t)^{2}\|e(t)\|^{2}\right) e(t)
$$

where $\alpha(s)=1 /(1-s)$ for $s \in[0,1)$. We choose $\varphi(t)=\left(4 e^{-2 t}+0.1\right)^{-1}=\varphi_{1}(t)$ for $t \geq 0$.

The simulation of the controllers (9) and (30) applied to (29) over the time interval $[0,10]$ is depicted in Fig. 8. It can be seen that for this example both controllers exhibit a nearly identical performance. 
Remark 3.1 A closer look at the simulations reveals that the controller performance of (9) differs from that of the controller presented in [8] for the example in Sect. 3.1, while it is practically identical for the example in Sect. 3.2. Since the different dimensions of input/output spaces ( $m=1$ compared to $m=2$ ) is probably not the reason here, the presumable cause seems to be the internal dynamics. System (26) has two-dimensional internal dynamics in Case $1(r=2)$ and one-dimensional internal dynamics in Case $2(r=3)$, while system (29) has trivial internal dynamics. This seems to suggest that the controllers exhibit a different behaviour in the presence of non-trivial internal dynamics.

\subsection{A nonlinear system with dead-zone input}

To demonstrate that the controller (9) can achieve asymptotic tracking and is feasible when the control direction is unknown, we treat a system with dead-zone input and also investigate the case wherein $\dot{y}_{\text {ref }}(t)$ is not available for feedback. Specifically, we consider

$$
\left.\begin{array}{l}
\dot{\xi}_{1}(t)=\left(1+\xi_{1}(t)^{2}\right) \xi_{2}(t), \\
\dot{\xi}_{2}(t)=\alpha_{1} \xi_{1}(t)+\alpha_{2} \xi_{2}(t)+\alpha_{3} \eta(t)+\beta(u(t)), \\
\dot{\eta}(t)=-\eta(t)^{2}\left(\alpha_{4} \xi_{1}(t)+\alpha_{5} \xi_{2}(t)+\eta(t)\right), \\
\left(\xi_{1}(0), \xi_{2}(0), \eta(0)\right)=\left(\xi_{1}^{0}, \xi_{2}^{0}, \eta^{0}\right) \in \mathbb{R}^{3}
\end{array}\right\} \text { with output } y(t)=\xi_{1}(t)
$$

with real parameters $\alpha_{i} \in \mathbb{R}, i=1, \ldots, 5$, and with a dead-zone input function $\beta$ as in Sect. 2.4. We show that system (31) belongs to the class of systems $\mathscr{N}^{1,2}$. Introducing the function

$$
g: \mathbb{R}^{2} \times \mathbb{R} \rightarrow \mathbb{R},(x, z)=\left(x_{1}, x_{2}, z\right) \mapsto-z^{2}\left(\alpha_{4} x_{1}+\alpha_{5}\left(1+x_{1}^{2}\right)^{-1} x_{2}+z\right),
$$

and writing $y_{1}(t)=\xi_{1}(t)=y(t)$ and $y_{2}(t)=\left(1+\xi_{1}(t)^{2}\right) \xi_{2}(t)=\dot{y}(t)$, the third equation in (31) may be expressed in the form

$$
\dot{\eta}(t)=g\left(y_{1}(t), y_{2}(t), \eta(t)\right), \quad \eta(0)=\eta^{0}
$$

which, viewed in isolation as a system with independent inputs $\left(y_{1}, y_{2}\right)$, generates a controlled flow $\Xi$. In particular, for $\eta^{0} \in \mathbb{R}$ and $\left(y_{1}, y_{2}\right) \in L_{\text {loc }}^{\infty}\left(\mathbb{R}_{\geq 0}, \mathbb{R}^{2}\right)$, the initialvalue problem (32) has unique maximal solution $\eta(\cdot)=\Xi\left(\cdot ; y_{1}, y_{2}, \eta^{0}\right):[0, \omega) \rightarrow$ $\mathbb{R}, 0<\omega \leq \infty$. Also, writing $\alpha:=\left|\alpha_{4}\right|+\left|\alpha_{5}\right|$, we have

$$
\forall(x, z) \in \mathbb{R}^{2} \times \mathbb{R}: z g(x, z) \leq-z^{4}+\alpha|z|^{3}\|x\| \leq-\frac{1}{4} z^{4}+\frac{1}{4}(\alpha\|x\|)^{4},
$$

wherein Young's inequality has been used. Therefore, $V: z \mapsto \frac{1}{2} z^{2}$ is an ISSLyapunov function for system (32) which, in consequence, is input-to-state stable, see [53, Rem. 2.4 and Lem. 2.14]. Therefore, for all $c_{0}>0$, there exists $c_{1}>0$ such 
that for all $\eta^{0} \in \mathbb{R}$ and all $\left(y_{1}, y_{2}\right) \in L_{\text {loc }}^{\infty}\left(\mathbb{R}_{\geq 0}, \mathbb{R}^{2}\right)$ we have

$$
\left\|\eta^{0}\right\|+\operatorname{ess}_{\sup _{t \geq 0}}\left\|\left(y_{1}(t), y_{2}(t)\right)\right\| \leq c_{0} \quad \Longrightarrow \quad \sup _{t \geq 0}\left\|\Xi\left(t ; y_{1}, y_{2}, \eta^{0}\right)\right\| \leq c_{1} \text {. }
$$

The above property ensures that solutions of (32) are globally defined: specifically, for each $\eta^{0} \in \mathbb{R}$ and $\left(y_{1}, y_{2}\right) \in L_{\text {loc }}^{\infty}\left(\mathbb{R}_{\geq 0}, \mathbb{R}^{2}\right)$, the unique maximal solution of (32) has interval of existence $[0, \infty)$. Therefore, with each fixed $\eta^{0} \in \mathbb{R}$, we may associate an operator

$$
\mathbf{T}: C\left(\mathbb{R}_{\geq 0}, \mathbb{R}^{2}\right) \rightarrow L_{\mathrm{loc}}^{\infty}\left(\mathbb{R}_{\geq 0}, \mathbb{R}^{3}\right), \quad\left(y_{1}, y_{2}\right) \mapsto\left(y_{1}, y_{2}, \Xi\left(\cdot ; y_{1}, y_{2}, \eta^{0}\right)\right) .
$$

Clearly, $\mathbf{T}$ is causal, i.e. (TP1) holds. Moreover, the above ISS property of $\Xi$ ensures that properties (TP2) and (TP3) hold. Therefore, $\mathbf{T} \in \mathbb{T}_{0}^{2,3}$. Defining

$$
f: \mathbb{R}^{4} \rightarrow \mathbb{R},\left(x_{1}, x_{2}, z, u\right) \mapsto \frac{2 x_{1} x_{2}^{2}}{1+x_{1}^{2}}+\alpha_{2} x_{2}+\left(1+x_{1}^{2}\right)\left(\alpha_{1} x_{1}+\alpha_{3} z+\beta(u)\right),
$$

it is readily verified that (31) is equivalent to

$$
\ddot{y}(t)=f(\mathbf{T}(y, \dot{y})(t), u(t)), \quad y(0)=\xi_{1}^{0}, \dot{y}(0)=\left(1+\left(\xi_{1}^{0}\right)^{2}\right) \xi_{2}^{0} .
$$

Furthermore, by applying the findings of Sects. 2.3 and 2.4 we have that $f$ satisfies the high-gain property and hence $(0, f, \mathbf{T}) \in \mathscr{N}^{1,2}$. Therefore, feasibility of funnel control follows from Theorem 1.9.

For the simulation, we (randomly) select

$$
\alpha_{1}=\alpha_{3}=\alpha_{5}=1 \text { and } \alpha_{2}=-\alpha_{4}=-2,
$$

and the dead-zone input function as

$$
\beta: \mathbb{R} \rightarrow \mathbb{R}, \quad v \mapsto \begin{cases}v-1, & v \geq 1 \\ 0, & -1<v<1 \\ v+1, & v \leq-1\end{cases}
$$

The initial values are chosen as $\xi_{1}(0)=\xi_{2}(0)=\eta(0)=0$ and the reference signal is $y_{\mathrm{ref}}: t \mapsto \cos t$. For the funnel controller (9) we choose the design parameters $\alpha: s \mapsto 1 /(1-s)$ and $N: s \mapsto s \sin s$; the latter choice is based on the assumption that the exact shape of $\beta$ (and in particular the control direction) is unknown to the controller.

We consider two different cases: If information of the instantaneous signals $\dot{y}_{\text {ref }}(t)$ are available to the controller, then we choose $\hat{r}=2=r$ and an unbounded funnel function $\varphi: t \mapsto t^{2}$. If information of $\dot{y}_{\text {ref }}(t)$ is not available, then we choose $\hat{r}=1<2$ and a bounded funnel function $\varphi: t \mapsto\left(2 e^{-t}+0.01\right)^{-1}$.

The simulation of the controller (9) applied to (31) in the cases $\hat{r}=1$ and $\hat{r}=2$ is depicted in Fig. 9. The "jumps" in the input $u$ are due to the dead-zone induced by the 


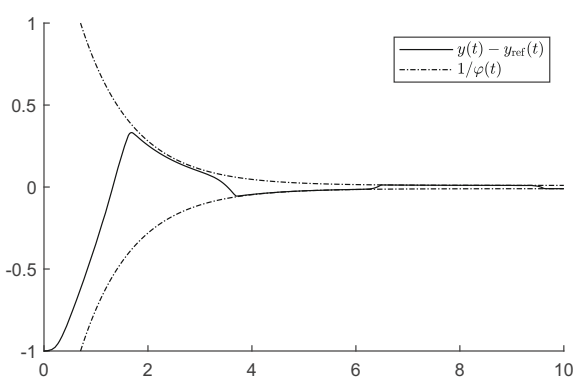

a Funnel and tracking error for $\hat{\mathrm{r}}=1$

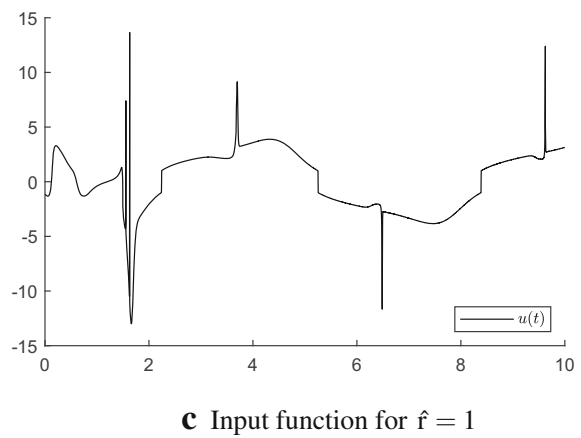

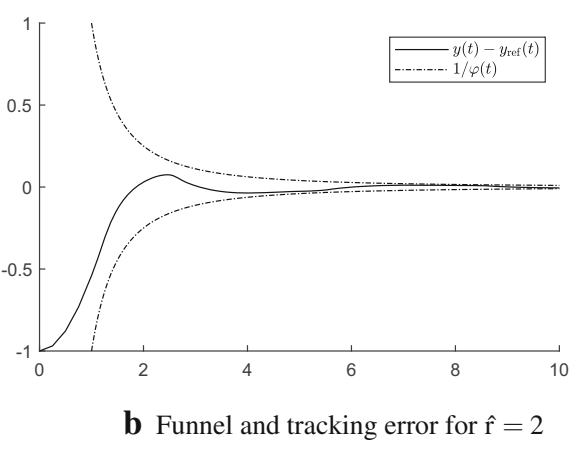

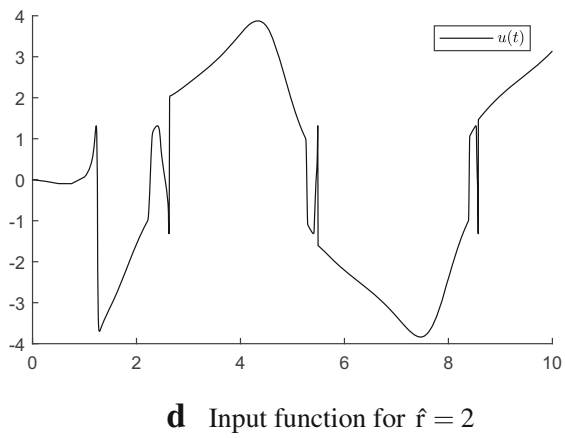

Fig. 9 Simulation of system (31) under control (9) in the cases $\hat{r}=1$ and $\hat{r}=2$

function $\beta$. Comparing Fig. $9 \mathrm{c}$ and $\mathrm{d}$ a degradation in performance may be observed. However, this is not surprising in view of the enhanced information available for feedback in case $\hat{r}=2$. We may also observe, that in the latter case asymptotic tracking is achieved.

\section{Conclusion}

An asymptotic and non-asymptotic tracking control objective has been achieved for a large class of nonlinear systems with "higher relative degree" described by functional differential equations that satisfy a high-gain property. A feedback strategy has been developed which is simple in the sense of funnel control and as "simple" as one may expect for higher relative degree. We believe that the present contribution is somehow "definitive" in the context of funnel control for nonlinear systems whose internal dynamics satisfy a BIBO property (viewed as a generalization of the minimum phase condition for linear systems). First results on funnel control for systems which are not minimum phase are given in [5] for uncertain linear systems and in [7] for a nonlinear robotic manipulator.

In the present paper, we did not treat funnel control for systems described by partial differential equations. This is however, a very important field and in fact very differ- 
ent. On the one hand, there are systems which have a well-defined relative degree and exhibit infinite-dimensional internal dynamics, see e.g. [11]. Such systems are susceptible to funnel control with the control laws presented in the present paper; for instance, a linearized model of a moving water tank, where sloshing effects appear, is discussed in [10]. On the other hand, not even every linear infinite-dimensional system has a well-defined relative degree, in which case the results presented here cannot be applied. For such systems, the feasibility of funnel control has to be investigated directly for the (nonlinear) closed-loop system, see e.g. [48] for a boundary controlled heat equation, [47] for a general class of boundary control systems, [6] for the monodomain equations (which represents defibrillation processes of the human heart) and [4] for the Fokker-Planck equation corresponding to the Ornstein-Uhlenbeck process.

One important problem remains: non-derivative funnel control, that is, when only the output $y$ is available for feedback, but not its first $r-1$ derivatives $\dot{y}, \ldots, y^{(r-1)}$. First results on this have been obtained in [31,32] using a backstepping approach. However, these results necessitate a level of controller complexity which, on the evidence of numerical simulation, can lead to practical performance drawbacks. An attempt to overcome these backstepping-induced drawbacks through the adoption of precompensators can be found in $[15,16]$ but only for systems with relative degree at most three: the higher relative degree case remains open, even in the context of single-input, single-output linear systems with positive high-frequency gain and asymptotically stable zero dynamics.

Funding Open Access funding enabled and organized by Projekt DEAL.

Open Access This article is licensed under a Creative Commons Attribution 4.0 International License, which permits use, sharing, adaptation, distribution and reproduction in any medium or format, as long as you give appropriate credit to the original author(s) and the source, provide a link to the Creative Commons licence, and indicate if changes were made. The images or other third party material in this article are included in the article's Creative Commons licence, unless indicated otherwise in a credit line to the material. If material is not included in the article's Creative Commons licence and your intended use is not permitted by statutory regulation or exceeds the permitted use, you will need to obtain permission directly from the copyright holder. To view a copy of this licence, visit http://creativecommons.org/licenses/by/4.0/.

\section{Appendix A: Proofs}

Proof of Theorem 1.9 For $k=1, \ldots, r$, we define

$$
\begin{aligned}
& \pi_{k}: \mathbb{R}_{\geq 0} \times \mathbb{R}^{r m} \rightarrow \mathbb{R}^{k m}, \\
& (t, \xi)=\left(t, \xi_{1}, \ldots, \xi_{r}\right) \mapsto\left\{\begin{array}{c}
\varphi(t)\left(\xi_{1}-y_{\mathrm{ref}}(t), \ldots, \xi_{k}-y_{\mathrm{ref}}^{(k-1)}(t)\right), \quad k=1, \ldots, \hat{r}, \\
\varphi(t)\left(\xi_{1}-y_{\mathrm{ref}}(t), \ldots, \xi_{\hat{r}}-y_{\mathrm{ref}}^{(\hat{r}-1)}(t), \xi_{\hat{r}+1}, \ldots, \xi_{k}\right), \\
k=\hat{r}+1, \ldots, r .
\end{array}\right.
\end{aligned}
$$

The proof now proceeds in several steps. 
Step 1. We recast the feedback-controlled system in the form of an initial-value problem to which a variant of an extant existence theory applies. Set $n=r m$ and

$$
\mathscr{D}:=\left\{(t, \xi) \in \mathbb{R}_{\geq 0} \times \mathbb{R}^{n} \mid \pi_{r}(t, \xi) \in \mathscr{D}_{r}\right\}
$$

which is non-empty and relatively open, and define $\rho: \mathscr{D} \rightarrow \mathscr{B}$ by $\rho:=\rho_{r} \circ \pi_{r}$. Introducing the function $F: \mathscr{D} \times \mathbb{R}^{q} \rightarrow \mathbb{R}^{n}$ given by

$$
(t, \xi, \eta)=\left(t, \xi_{1}, \ldots, \xi_{r}, \eta\right) \mapsto F(t, \xi, \eta):=\left(\begin{array}{c}
\xi_{2} \\
\vdots \\
\xi_{r} \\
f\left(d(t), \eta,(N \circ \alpha)\left(\|\rho(t, \xi)\|^{2}\right) \rho(t, \xi)\right)
\end{array}\right)
$$

and writing

$$
x(t)=\left(\begin{array}{c}
y(t) \\
\vdots \\
y^{(r-1)}(t)
\end{array}\right)
$$

we see that the (formal) control (9) may be expressed as

$$
u(t)=(N \circ \alpha)\left(\|\rho(t, x(t))\|^{2}\right) \rho(t, x(t)) .
$$

The feedback-controlled initial-value problem (1) and (9) may now be formulated as

$$
\dot{x}(t)=F(t, x(t), \mathbf{T}(x)(t)),\left.\quad x\right|_{[-h, 0]}=x^{0} \in C\left([-h, 0], \mathbb{R}^{n}\right),
$$

where

$$
x^{0}(t):=\left(\begin{array}{c}
y^{0}(t) \\
\vdots \\
\left(y^{0}\right)^{(r-1)}(t)
\end{array}\right), \quad t \in[-h, 0]
$$

A continuous function $x \in C\left(I, \mathbb{R}^{n}\right)$ on an interval of the form $I=[-h, \tilde{\omega}], 0<$ $\tilde{\omega}<\infty$, or of the form [-h, $\left[-, 0<\omega \leq \infty\right.$, is a solution of (33), if $\left.x\right|_{[-h, 0]}=x^{0}$, $(t, x(t)) \in \mathscr{D}$ for all $t \in I \backslash[-h, 0)$ and

$$
\forall t \in I, t \geq 0: x(t)=x\left(t_{0}\right)+\int_{0}^{t} F(s, x(s), \mathbf{T}(x)(s)) \mathrm{d} s .
$$

A solution is maximal, if it has no right extension that is also a solution. Since $\mathbf{T}$ is an operator with domain $C\left([-h, \infty), \mathbb{R}^{n}\right)$, some care is required in interpreting the above notion of a solution $x \in C\left(I, \mathbb{R}^{n}\right)$ when $I$ is a bounded interval of the form $I=[-h, \tilde{\omega}]$ 
or $I=[-h, \omega)$. Let $I$ be any such interval and write $J:=I \backslash[-h, 0)$. Let $x \in C\left(I, \mathbb{R}^{n}\right)$ and, for each $\tau \in J$, define $x_{\tau} \in C\left([-h, \infty), \mathbb{R}^{n}\right)$ by

$$
x_{\tau}(t):=\left\{\begin{array}{l}
x(t), \quad t \in[-h, \tau] \\
x(\tau), \quad t>\tau .
\end{array}\right.
$$

With $\mathbf{T} \in \mathbb{T}_{h}^{n, q}$ we may associate $\tilde{\mathbf{T}}: C\left(I, \mathbb{R}^{n}\right) \rightarrow L_{\text {loc }}^{\infty}\left(J, \mathbb{R}^{q}\right)$ defined by the property

$$
\forall \tau \in J:\left.\tilde{\mathbf{T}}(x)\right|_{[0, \tau]}=\left.\mathbf{T}\left(x_{\tau}\right)\right|_{[0, \tau]} .
$$

The causality property (P1) of $\mathbf{T} \in \mathbb{T}_{h}^{n, q}$ ensures that $\tilde{\mathbf{T}}$ is well defined. Replacing $\mathbf{T}$ by $\tilde{\mathbf{T}}$ in (34), we arrive at the correct interpretation of a solution. However, for simplicity, we will not distinguish notationally between an operator $\mathbf{T} \in \mathbb{T}_{h}^{n, q}$ and its "localization" $\tilde{\mathbf{T}}$.

It is readily verified that $F$ has the following properties: If $I \subset \mathbb{R}_{\geq 0}$ is a compact interval and $K_{n} \subset \mathbb{R}^{n}, K_{q} \subset \mathbb{R}^{q}$ are compact with $I \times K_{n} \subset \mathscr{D}$, then

(a) $F(t, \cdot, \cdot): K_{n} \times K_{q} \rightarrow \mathbb{R}^{n}$ is continuous for all $t \in I$;

(b) $F(\cdot, v, w): I \rightarrow \mathbb{R}^{n}$ is measurable for all $(v, w) \in K_{n} \times K_{q}$;

(c) there exists $\hat{f} \in(0, \infty)$ such that $\|F(t, v, w)\| \leq \hat{f}$ for almost all $t \in I$ and all $(v, w) \in K_{n} \times K_{q}$.

Invoking (11), we see that $\left(0, x^{0}(0)\right) \in \mathscr{D}$. An application of a variant (a straightforward modification tailored to the current context) of [28, Thm. B.1] yields the existence of a maximal solution $x:[-h, \omega) \rightarrow \mathbb{R}^{n}, 0<\omega \leq \infty$, of (33) and so

$$
\mathscr{G}=\operatorname{graph}\left(\left.x\right|_{[0, \omega)}\right) \subset \mathscr{D} \text {. }
$$

Moreover, the closure of $\mathscr{G}$ is not a compact subset of $\mathscr{D}$.

Step 2. Before embarking on the proof proper, we record some preliminary observations and definitions. Since $(t, x(t)) \in \mathscr{D}$ for all $t \in[0, \omega)$, we have $\pi_{k}(t, x(t)) \in$ $\mathscr{D}_{k}=\operatorname{dom}\left(\rho_{k}\right), k=1, \ldots, r$. Introduce continuous functions

$$
e_{k}:[0, \omega) \rightarrow \mathscr{B}, \quad \alpha_{k}:[0, \omega) \rightarrow[1, \infty), \quad \gamma_{k}:[0, \omega) \rightarrow \mathbb{R}^{m}, \quad k=1, \ldots, r,
$$

given by

$e_{k}(t):=\left(\rho_{k} \circ \pi_{k}\right)(t, x(t)), \quad \alpha_{k}(t):=\alpha\left(\left\|e_{k}(t)\right\|^{2}\right), \quad \gamma_{k}(t):=\gamma\left(e_{k}(t)\right)=\alpha_{k}(t) e_{k}(t)$,

where $\gamma$ is given by (7), and, for later notational consistency, we also write $\gamma_{0}(\cdot):=0$. Clearly,

$$
\forall k=1, \ldots, r \forall t \in[0, \omega):\left\|e_{k}(t)\right\|<1 .
$$

In particular, for $k=1$ we have $\left\|e_{1}(t)\right\|=\varphi(t)\|e(t)\|<1$ for all $t \in[0, \omega)$ and so the tracking error $e(\cdot)=y(\cdot)-y_{\text {ref }}(\cdot)$ evolves in the funnel $\mathscr{F}_{\varphi}$. 
Observe that the continuous control function $u$ may be expressed as

$$
u(t)=N\left(\alpha_{r}(t)\right) e_{r}(t), \quad t \in[0, \omega),
$$

and, for all $t \in[0, \omega)$ and $k=1, \ldots, r$, we have by definition of $\rho_{k}$ in (8)

$$
e_{k}(t)-\gamma_{k-1}(t)= \begin{cases}\varphi(t) e^{(k-1)}(t), & \text { if } k \leq \hat{r} \\ \varphi(t) y^{(k-1)}(t), & \text { otherwise. }\end{cases}
$$

We also record that

$$
\dot{\alpha}_{k}(t)=-2 \alpha^{\prime}\left(\left\|e_{k}(t)\right\|^{2}\right)\left\langle e_{k}(t), \dot{e}_{k}(t)\right\rangle \text { for a.a. } t \in[0, \omega), \quad k=1, \ldots, r .
$$

Define functions $\psi_{k}:[0, \infty) \rightarrow \mathbb{R}^{m}, k=1, \ldots, r$, as follows

$$
\begin{aligned}
& \hat{r}=r \Longrightarrow \psi_{k}(\cdot):=0, k=1, \ldots, r \\
& \hat{r}<r \Longrightarrow \psi_{k}(t):= \begin{cases}0, & \text { if } k<\hat{r} \\
-\varphi(t) y_{\text {ref }}^{(\hat{r})}(t), & \text { if } k=\hat{r} \\
\dot{\varphi}(t) y_{\mathrm{ref}}^{(k-1)}, & \text { if } \hat{r}<k \leq r-1 \\
\dot{\varphi}(t) y_{\mathrm{ref}}^{(r-1)}(t)+\varphi(t) y_{\text {ref }}^{(r)}(t), & \text { if } k=r .\end{cases}
\end{aligned}
$$

By choice of the design parameters as in (6), $\varphi$ is bounded (and so $\dot{\varphi}$ is essentially bounded by the definition of $\Phi$ ) if $\hat{r}<r$. Therefore, we may infer the existence of $\psi^{*} \in \mathbb{R}$ (with $\psi^{*}=0$ if $\hat{r}=r$ ) such that

$$
\left\|\psi_{k}(t)\right\| \leq \psi^{*} \text { for a.a. } t \in[0, \infty), k=1, \ldots, r .
$$

Observe that, for almost all $t \in[0, \omega)$,

$$
\left.\begin{array}{l}
\dot{e}_{k}(t)=\dot{\varphi}(t) e^{(k-1)}(t)+e_{k+1}(t)-\gamma_{k}(t)+\dot{\gamma}_{k-1}(t)+\psi_{k}(t), k=1, \ldots, r-1 \\
\dot{e}_{r}(t)=\dot{\varphi}(t) e^{(r-1)}(t)+\varphi(t) e^{(r)}(t)+\dot{\gamma}_{r-1}(t)+\psi_{r}(t)
\end{array}\right\}
$$

which, if $r=1=\hat{r}$, collapses to the tautology: $\dot{e}_{1}(t)=(\varphi e)^{(1)}(t)$ for a.a. $t \in[0, \omega)$. Arbitrarily fix $\tau \in(0, \omega)$. By continuity, there exists $\theta \in(0, \infty)$ such that

$$
\forall t \in[0, \tau]:(1+\varphi(t)) \sum_{k=1}^{r}\left\|e^{(k-1)}(t)\right\| \leq \theta
$$

and so, by properties of $\Phi$, there exists $c>0$ such that

$$
\left\|\dot{\varphi}(t) e^{(k-1)}(t)\right\| \leq c(1+\varphi(t))\left\|e^{(k-1)}(t)\right\| \leq c \theta \text { for a.a. } t \in[0, \tau], \quad k=1, \ldots, r .
$$


Again by properties of $\Phi$, the following are well defined:

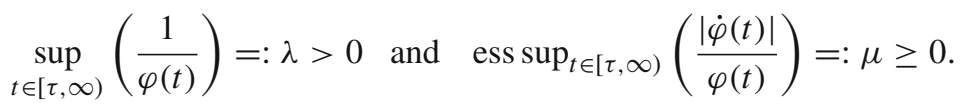

For $k \in\{1, \ldots, r\}$ and invoking (35), (37) and (39), we find

$$
\text { (a) } \begin{aligned}
\left\|\dot{\varphi}(t) e^{(k-1)}(t)\right\| & \leq \mu\left\|\varphi(t) e^{(k-1)}(t)\right\| \leq \mu\left(1+\left\|\gamma_{k-1}(t)\right\|\right) \\
& \text { for a.a. } t \in[\tau, \omega), \quad \text { if } k \leq \hat{r} \\
\text { (b) }\left\|\dot{\varphi}(t) e^{(k-1)}(t)\right\| & \leq \mu\left\|\varphi(t) y^{(k-1)}(t)\right\|+\left\|\dot{\varphi}(t) y_{\text {ref }}^{(k-1)}\right\| \\
& \leq \mu\left(1+\left\|\gamma_{k-1}(t)\right\|\right)+\psi^{*} \\
& \text { for a.a. } t \in[\tau, \omega), \text { if } k>\hat{r}
\end{aligned}
$$

and so, a fortiori, we have

$$
\left\|\dot{\varphi}(t) e^{(k-1)}(t)\right\| \leq \mu\left(1+\left\|\gamma_{k-1}(t)\right\|\right)+\psi^{*} \text { for a.a. } t \in[\tau, \omega), k=1, \ldots, r .
$$

We complete the preliminaries by writing

$$
\hat{\varepsilon}_{k}:=\max _{t \in[0, \tau]}\left\|e_{k}(t)\right\|^{2}<1, \quad k=1, \ldots, r
$$

Step 3. Assume that $r \geq 2$, otherwise proceed to Step 5. Let $\varepsilon_{1}$ be the unique point of $(0,1)$ such that $\varepsilon_{1} \alpha\left(\varepsilon_{1}\right)=1+\mu+2 \psi^{*}$ and $\varepsilon_{1}^{*}:=\max \left\{\hat{\varepsilon}_{1}, \varepsilon_{1}\right\}<1$. We will show that

$$
\forall t \in[0, \omega):\left\|e_{1}(t)\right\|^{2} \leq \varepsilon_{1}^{*} .
$$

Suppose that this claim is false. Then $\left\|e_{1}(s)\right\|^{2}>\varepsilon_{1}^{*}$ for some $s \in(0, \omega)$. Since $\left\|e_{1}(t)\right\|^{2} \leq \hat{\varepsilon}_{1} \leq \varepsilon_{1}^{*}$ for all $t \in[0, \tau]$, we have $\tau<s$ and so we may define

$$
\sigma:=\max \left\{t \in[\tau, s) \mid\left\|e_{1}(t)\right\|^{2}=\varepsilon_{1}^{*}\right\}
$$

Clearly,

$$
\forall t \in[\sigma, s]: \varepsilon_{1} \leq \varepsilon_{1}^{*} \leq\left\|e_{1}(t)\right\|^{2},
$$

whence, by monotonicity of $\alpha$,

$$
\forall t \in[\sigma, s]: \alpha\left(\varepsilon_{1}\right) \leq \alpha\left(\left\|e_{1}(t)\right\|^{2}\right)=\alpha_{1}(t)
$$

Therefore,

$$
\forall t \in[\sigma, s]: \alpha_{1}(t)\left\|e_{1}(t)\right\|^{2} \geq \varepsilon_{1} \alpha\left(\varepsilon_{1}\right)=1+\mu+2 \psi^{*}
$$


which, by the first of relations (40) in conjunction with (35) and (43) (and recalling $\left.\gamma_{0}(\cdot)=0\right)$, gives

$$
\begin{aligned}
\frac{1}{2} \frac{\mathrm{d}}{\mathrm{d} t}\left\|e_{1}(t)\right\|^{2} & =\left\langle e_{1}(t), \dot{e}_{1}(t)\right\rangle \\
& =\left\langle\dot{\varphi}(t) e_{1}(t), e(t)\right\rangle+\left\langle e_{1}(t), e_{2}(t)\right\rangle-\alpha_{1}(t)\left\|e_{1}(t)\right\|^{2}+\left\langle e_{1}(t), \psi_{1}(t)\right\rangle \\
& <1+\mu+2 \psi^{*}-\alpha_{1}(t)\left\|e_{1}(t)\right\|^{2} \leq 0
\end{aligned}
$$

for almost all $t \in[\sigma, s]$ and so $\left\|e_{1}(s)\right\|^{2}<\left\|e_{1}(\sigma)\right\|^{2}$, whence the contradiction

$$
\varepsilon_{1}^{*}<\left\|e_{1}(s)\right\|^{2}<\left\|e_{1}(\sigma)\right\|^{2}=\varepsilon_{1}^{*} .
$$

Therefore (44) holds.

Step 4. For notational convenience, write

$$
W_{1}:=W^{1, \infty}([0, \omega), \mathbb{R}) \text { and } W_{m}:=W^{1, \infty}\left([0, \omega), \mathbb{R}^{m}\right) .
$$

We show by induction that

$$
\left(\alpha_{k}, e_{k}, \gamma_{k}\right) \in W_{1} \times W_{m} \times W_{m} \text { for } k=1, \ldots, r-1
$$

This step is vacuous in the case $r=1$. Let $k=1$. By (44), we see that $e_{1}$ is bounded by $\sqrt{\varepsilon_{1}^{*}}, \alpha_{1}$ is bounded by $\alpha\left(\varepsilon_{1}^{*}\right)$ and that $\gamma_{1}$ is bounded by $\sqrt{\varepsilon_{1}^{*}} \alpha\left(\varepsilon_{1}^{*}\right)$. Recalling that $\gamma_{0}(\cdot)=0$, essential boundedness of $\dot{e}_{1}$ follows by the first of relations (40) together with (35), (39), (42), (43). Invoking (38), we may conclude essential boundedness of $\dot{\alpha}_{1}$. Essential boundedness of $\dot{\gamma}_{1}=\alpha_{1} \dot{e}_{1}+\dot{\alpha}_{1} e_{1}$ then follows. Therefore, $\left(\alpha_{1}, e_{1}, \gamma_{1}\right) \in W_{1} \times W_{m} \times W_{m}$.

Now assume that $k \in\{2, \ldots, r-1\}$ and

$$
\left(\alpha_{j}, e_{j}, \gamma_{j}\right) \in W_{1} \times W_{m} \times W_{m}, \quad j=1, \ldots, k-1
$$

Set

$$
\beta:=\max \left\{\psi^{*}, \sup _{t \in[0, \omega)}\left\|\gamma_{k-1}(t)\right\|, \operatorname{ess} \sup _{t \in[0, \omega)}\left\|\dot{\gamma}_{k-1}(t)\right\|\right\}<\infty
$$

By (35), (40) and (43), we have

$$
\begin{aligned}
\left\langle e_{k}(t), \dot{e}_{k}(t)\right\rangle= & \dot{\varphi}(t)\left\langle e_{k}(t), e^{(k-1)}(t)\right\rangle+\left\langle e_{k}(t), e_{k+1}(t)\right\rangle \\
& +\left\langle e_{k}(t),\left(\dot{\gamma}_{k-1}(t)+\psi_{k}(t)\right)\right\rangle-\alpha_{k}(t)\left\|e_{k}(t)\right\|^{2} \\
< & 1+3 \beta+\mu(1+\beta)-\alpha_{k}(t)\left\|e_{k}(t)\right\|^{2}
\end{aligned}
$$

for almost all $t \in[\tau, \omega)$. Let $\varepsilon_{k}$ be the unique point of $(0,1)$ such that $\varepsilon_{k} \alpha\left(\varepsilon_{k}\right)=$ $1+3 \beta+\mu(1+\beta)$ and define

$$
\varepsilon_{k}^{*}:=\max \left\{\hat{\varepsilon}_{k}, \varepsilon_{k}\right\}>0 .
$$


We first show that

$$
\forall t \in[0, \omega):\left\|e_{k}(t)\right\|^{2} \leq \varepsilon_{k}^{*}
$$

by the contradiction argument of Step 3 (mutatis mutandis). Suppose that (48) is false. Then $\left\|e_{k}(s)\right\|^{2}>\varepsilon_{k}^{*}$ for some $s \in(0, \omega)$. Since $\left\|e_{k}(t)\right\|^{2} \leq \hat{\varepsilon}_{k} \leq \varepsilon_{k}^{*}$ for all $t \in[0, \tau]$, we have $\tau<s$ and so we may define $\sigma:=\max \left\{t \in[\tau, s) \mid\left\|e_{k}(t)\right\|^{2}=\varepsilon_{k}^{*}\right\}$. The counterpart of (45) now follows:

$$
\forall t \in[\sigma, s]: \alpha_{k}(t)\left\|e_{k}(t)\right\|^{2} \geq \varepsilon_{k} \alpha\left(\varepsilon_{k}\right)=1+3 \beta+\mu(1+\beta)
$$

which, in conjunction with (47), gives $\frac{1}{2} \frac{\mathrm{d}}{\mathrm{d} t}\left\|e_{k}(t)\right\|^{2}<0$ for almost all $t \in[\sigma, s]$, whence the contradiction

$$
\varepsilon_{k}^{*}<\left\|e_{k}(s)\right\|^{2}<\left\|e_{k}(\sigma)\right\|^{2}=\varepsilon_{k}^{*}
$$

Therefore, (48) holds which, in turn, implies that $\alpha_{k}$ is bounded (by $\alpha\left(\varepsilon_{k}^{*}\right)$ ) and that $\gamma_{k}=\alpha_{k} e_{k}$ is bounded (by $\sqrt{\varepsilon_{k}^{*}} \alpha\left(\varepsilon_{k}^{*}\right)$ ). By boundedness of $e_{k+1}, \gamma_{k}$ and essential boundedness of $\dot{\gamma}_{k-1}$, it follows from (40), together with (42) and (43), that $\dot{e}_{k}$ is essentially bounded and so $e_{k} \in W_{m}$. Invoking (38), we may now infer essential boundedness of $\dot{\alpha}_{k}$. Therefore, $\alpha_{k} \in W_{1}$. Finally, since $\dot{\gamma}_{k}=\alpha_{k} \dot{e}_{k}+\dot{\alpha}_{k} e_{k}$, we have essential boundedness of $\dot{\gamma}_{k}$ and so $\gamma_{k} \in W_{m}$. In summary, we have shown that, for $k \in\{2, \ldots, r-1\}$,

$$
\left(\alpha_{j}, e_{j}, \gamma_{j}\right) \in W_{1} \times W_{m} \times W_{m}, j=1, \ldots, k-1 \Longrightarrow\left(\alpha_{k}, e_{k}, \gamma_{k}\right) \in W_{1} \times W_{m} \times W_{m}
$$

and so, by induction, we conclude (46).

Step 5. Our next goal is to prove boundedness of the solution $x$. Recalling that $y_{\text {ref }} \in W^{r, \infty}\left(\mathbb{R}_{\geq 0}, \mathbb{R}^{m}\right)$, it suffices to show that the output error $e$ and its derivatives $\dot{e}, \ldots, e^{(r-1)}$ are bounded on $[0, \omega)$. By $(41)$, we already know that

$$
\forall k=1, \ldots, r \forall t \in[0, \tau]:\left\|e^{(k-1)}(t)\right\| \leq \theta,
$$

and so it remains to show that $e^{(k-1)}$ is bounded on $[\tau, \omega), k=1, \ldots, r$. Since $\varphi(t) e(t)=e_{1}(t) \in \mathscr{B}$ for all $t \in[0, \omega)$, we have

$$
\forall t \in[\tau, \omega):\|e(t)\| \leq \frac{1}{\varphi(t)} \leq \lambda
$$

By boundedness of the functions $\gamma_{k}$ (Step 4), there exists $\gamma^{*}>0$ such that

$$
\forall k=2, \ldots, r \forall t \in[0, \omega):\left\|\gamma_{k-1}(t)\right\| \leq \gamma^{*}
$$

Let $k \in\{2, \ldots, r\}$. By (37), we have

$$
\forall t \in[\tau, \omega):\left\|e^{(k-1)}(t)\right\| \leq \lambda\left(1+\gamma^{*}\right)+\sup _{t \geq \tau}\left\|y_{\mathrm{ref}}^{(k-1)}(t)\right\|<\infty .
$$


This completes Step 5.

Step 6. We prove boundedness of $\alpha_{r}:[0, \omega) \rightarrow[1, \infty), t \mapsto \alpha\left(\left\|e_{r}(t)\right\|^{2}\right)$ together with an immediate consequence thereof:

$$
\exists \varepsilon_{r}^{*} \in(0,1) \forall t \in[0, \omega):\left\|e_{r}(t)\right\|^{2} \leq \varepsilon_{r}^{*} .
$$

By boundedness of $x$ (Step 5) and property (TP3) of the operator class $\mathbb{T}_{h}^{n, q}$, there exists compact $K_{q} \subset \mathbb{R}^{q}$ such that $\mathbf{T}(x)(t) \in K_{q}$ for almost all $t \in[0, \omega)$. Since $d \in$ $L^{\infty}\left(\mathbb{R}_{\geq 0}, \mathbb{R}^{p}\right)$, there exists compact $K_{p} \subset \mathbb{R}^{p}$ such that $d(t) \in K_{p}$ for almost all $t \in$ $[0, \omega)$. By the high-gain property, there exists $v^{*} \in(0,1)$ such that the continuous function

$$
\chi: \mathbb{R} \rightarrow \mathbb{R}, \quad s \mapsto \min \left\{\langle v, f(\delta, z,-s v)\rangle \mid(\delta, z, v) \in K_{p} \times K_{q} \times A_{m}\right\}
$$

is unbounded from above, where, for notational convenience, we have introduced the compact annulus

$$
A_{m}:=\left\{v \in \mathbb{R}^{m} \mid v^{*} \leq\|v\| \leq 1\right\} .
$$

Choose a real sequence $\left(s_{j}\right)$ such that the sequence $\left(\chi\left(s_{j}\right)\right)$ is unbounded, positive, and strictly increasing. By surjectivity and continuity of $N$, for every $a \in \mathbb{R}_{\geq 0}$ and every $b \in \mathbb{R}$, the set $\{\kappa>a \mid N(\kappa)=b\}$ is non-empty. Choose $\kappa_{1}>\alpha\left(\left(1-v^{*}\right)^{2}\right)+\alpha_{r}(0)$ such that $N\left(\kappa_{1}\right)=s_{1}$ and define the strictly increasing sequence $\left(\kappa_{j}\right)$ by the recursion

$$
\kappa_{j+1}:=\inf \left\{\kappa>\kappa_{j} \mid N(\kappa)=s_{j+1}\right\} .
$$

Observe that

$$
\lim _{j \rightarrow \infty} \chi\left(N\left(\kappa_{j}\right)\right)=\lim _{j \rightarrow \infty} \chi\left(s_{j}\right)=\infty
$$

Seeking a contradiction, suppose that $\alpha_{r}(\cdot)$ is not bounded. Then, since $\kappa_{j+1}>\kappa_{1}>$ $\alpha_{r}(0)$ for all $j \in \mathbb{N}$, the sequence $\left(\tau_{j}\right)$ in $(0, \omega)$ defined by

$$
\tau_{j}=\inf \left\{t \in[0, \omega) \mid \alpha_{r}(t)=\kappa_{j+1}\right\}, \quad j \in \mathbb{N}_{0},
$$

is well-defined and strictly increasing with $N\left(\alpha_{r}\left(\tau_{j}\right)\right)=N\left(\kappa_{j+1}\right)=s_{j+1}$ for each $j \in$ $\mathbb{N}_{0}$. Now, define the sequence $\left(\sigma_{j}\right)$ in $(0, \omega)$ by

$$
\sigma_{j}=\sup \left\{t \in\left[\tau_{j-1}, \tau_{j}\right] \mid \chi\left(N\left(\alpha_{r}(t)\right)\right)=\chi\left(s_{j}\right)\right\}, \quad j \in \mathbb{N} .
$$

Since the sequence $\left(\chi\left(s_{j}\right)\right)$ is strictly increasing, we have

$$
\forall j \in \mathbb{N}: \chi\left(N\left(\alpha_{r}\left(\sigma_{j}\right)\right)\right)=\chi\left(s_{j}\right)<\chi\left(s_{j+1}\right)=\chi\left(N\left(\alpha_{r}\left(\tau_{j}\right)\right)\right),
$$


and so

$$
\forall j \in \mathbb{N} \forall t \in\left(\sigma_{j}, \tau_{j}\right]: \sigma_{j}<\tau_{j} \text { and } \chi\left(N\left(\alpha_{r}\left(\sigma_{j}\right)\right)\right)=\chi\left(s_{j}\right)<\chi\left(N\left(\alpha_{r}(t)\right)\right) \text {. }
$$

Next, suppose that, for some $j \in \mathbb{N}$, there exists $t \in\left[\sigma_{j}, \tau_{j}\right]$ such that $e_{r}(t) \notin A_{m}$. We first show that $\alpha_{r}(t) \geq \kappa_{j}$. If $\alpha_{r}(t)<\kappa_{j}$, then $\alpha_{r}\left(\tau_{j}\right)=\kappa_{j+1}>\kappa_{j}$ and continuity of $\alpha_{r}$ imply that there exists $\tilde{t} \in\left(\sigma_{j}, \tau_{j}\right)$ such that $\alpha_{r}(\tilde{t})=\kappa_{j}$, thus

$$
\chi\left(N\left(\alpha_{r}(\tilde{t})\right)\right)=\chi\left(N\left(\kappa_{j}\right)\right)=\chi\left(s_{j}\right)
$$

which contradicts the definition of $\sigma_{j}$. Therefore, $\alpha_{r}(t) \geq \kappa_{j}$ which, together with the supposition $\left\|e_{r}(t)\right\|<1-v^{*}$, leads to the contradiction:

$$
\alpha\left(\left(1-v^{*}\right)^{2}\right)<\kappa_{1} \leq \kappa_{j} \leq \alpha_{r}(t)=\alpha\left(\left\|e_{r}(t)\right\|^{2}\right)<\alpha\left(\left(1-v^{*}\right)^{2}\right) .
$$

Therefore,

$$
\forall j \in \mathbb{N} \forall t \in\left[\sigma_{j}, \tau_{j}\right]: e_{r}(t) \in A_{m},
$$

which, in conjunction with the facts that $d(t) \in K_{p}$ and $(\mathbf{T} x)(t) \in K_{q}$ for almost all $t \in[0, \omega)$ and invoking (51), yields

$$
\begin{aligned}
& \left\langle e_{r}(t), f(d(t),(\mathbf{T} x)(t), u(t))\right\rangle \\
& \quad=-\left\langle-e_{r}(t), f\left(d(t),(\mathbf{T} x)(t),-N\left(\alpha_{r}(t)\right)\left(-e_{r}(t)\right)\right)\right\rangle \\
& \quad \leq-\min \left\{\left\langle v, f\left(\delta, z,-N\left(\alpha_{r}(t)\right) v\right)\right\rangle \mid(\delta, z, v) \in K_{p} \times K_{q} \times A_{m}\right\} \\
& \quad=-\chi\left(N\left(\alpha_{r}(t)\right)\right) \leq-\chi\left(s_{j}\right)
\end{aligned}
$$

for all $j \in \mathbb{N}$ and almost all $t \in\left[\sigma_{j}, \tau_{j}\right]$. By (42), (43) and (49),

$$
\left\|\dot{\varphi}(t) e^{(r-1)}(t)\right\| \leq c \theta+\mu\left(1+\gamma^{*}\right)+\psi^{*}=: \theta^{*} \text { for a.a. } t \in[0, \omega)
$$

Since $e^{(r)}(t)=f(d(t), \mathbf{T}(x)(t), u(t))-y_{\text {ref }}^{(r)}(t)$ for almost all $t \in[0, \omega)$ and recalling the last of relations (40), we have

$$
\dot{e}_{r}(t)=\varphi(t)\left(f(d(t), \mathbf{T}(x)(t), u(t))-y_{\mathrm{ref}}^{(r)}(t)\right)+\dot{\varphi}(t) e^{(r-1)}(t)+\dot{\gamma}_{r-1}(t)+\psi_{r}(t)
$$

for almost all $t \in[0, \omega)$. By (46), $\dot{\gamma}_{r-1}$ is essentially bounded and, since $y_{\text {ref }} \in$ $W^{r, \infty}\left(\mathbb{R}_{\geq 0}, \mathbb{R}^{m}\right)$, we have essential boundedness of $y_{\text {ref }}^{(r)}$. Write

$$
c_{1}:=\theta^{*}+\psi^{*}+\operatorname{ess~sup}_{t \in[0, \omega)}\left\|\dot{\gamma}_{r-1}(t)\right\| \quad \text { and } \quad c_{2}:=\operatorname{ess~sup}_{t \geq 0}\left\|y_{\text {ref }}^{(r)}(t)\right\| .
$$

Invoking (35), (39) and (53), we arrive at

$$
\frac{1}{2} \frac{\mathrm{d}}{\mathrm{d} t}\left\|e_{r}(t)\right\|^{2} \leq c_{1}-\varphi(t)\left(\chi\left(s_{j}\right)-c_{2}\right)
$$


for all $j \in \mathbb{N}$ and almost all $t \in\left[\sigma_{j}, \tau_{j}\right]$. By properties of $\varphi \in \Phi$ and noting that $\sigma_{1}>0$, we have $\inf _{t \in\left[\sigma_{1}, \infty\right)} \varphi(t)>0$. Since $\chi\left(s_{j}\right) \rightarrow \infty$ as $j \rightarrow \infty$, we may choose $j$ sufficiently large so that $c_{1}-\varphi(t)\left(\chi\left(s_{j}\right)-c_{2}\right)<0$ for almost all $t \in\left[\sigma_{j}, \tau_{j}\right]$, in which case we have $\left\|e_{r}\left(\tau_{j}\right)\right\|^{2}<\left\|e_{r}\left(\sigma_{j}\right)\right\|^{2}$ and so

$$
\alpha_{r}\left(\tau_{j}\right)=\alpha\left(\left\|e_{r}\left(\tau_{j}\right)\right\|^{2}\right)<\alpha\left(\left\|e_{r}\left(\sigma_{j}\right)\right\|^{2}\right)=\alpha_{r}\left(\sigma_{j}\right)
$$

which is impossible since, by definition of $\tau_{j}$, we have $\alpha_{r}(t)<\alpha_{r}\left(\tau_{j}\right)$ for all $t \in$ $\left[0, \tau_{j}\right)$. Therefore, our original supposition that $\alpha_{r}$ is unbounded is false. This proves (50) and completes the proof of Step 6.

Step 7. We prove Assertion (i) of the theorem. Recalling inequalities (44), (48) and (50) of Steps 3, 4 and 6, we have

$$
\left\|e_{k}(t)\right\| \leq \varepsilon:=\sqrt{\max \left\{\varepsilon_{1}^{*}, \ldots, \varepsilon_{r}^{*}\right\}}<1
$$

for all $t \in[0, \omega)$ and all $k=1, \ldots, r$. Define

$$
\widehat{\mathscr{D}}_{r}:=\left\{\left(\eta_{1}, \ldots, \eta_{r}\right) \in \mathbb{R}^{r m} \mid\left\|\rho_{k}\left(\eta_{1}, \ldots, \eta_{k}\right)\right\| \leq \varepsilon, k=1, \ldots, r\right\},
$$

which is evidently a compact subset of $\mathscr{D}_{r}$ as in (8). Since $e_{k}(t)=\left(\rho_{k} \circ \pi_{k}\right)(t, x(t))$ for all $t \in[0, \omega), k=1, \ldots, r$, it follows that $\pi_{r}(t, x(t)) \in \widehat{\mathscr{D}}_{r}$ for all $t \in[0, \omega)$. Suppose that $\omega<\infty$. Then

$$
\forall t \in[0, \omega):(t, x(t)) \in \widehat{\mathscr{D}}:=\left\{(s, \xi) \in[0, \omega] \times \mathbb{R}^{r m} \mid \pi_{r}(s, \xi) \in \widehat{\mathscr{D}}_{r}\right\} \subset \mathscr{D} .
$$

By compactness of $\widehat{\mathscr{D}}$ it follows that the closure of $\operatorname{graph}\left(\left.x\right|_{[0, \omega)}\right)$ is a compact subset of $\mathscr{D}$, which contradicts the findings of Step 1. Therefore, $\omega=\infty$.

Step 8. We complete the proof by establishing Assertions (ii), (iii) and (iv). Assertion(ii) is a direct consequence of Assertion(i) and the results of Steps 5 and 6. Recalling that $e_{1}=\varphi e$, we may infer Assertion(iii) from (54) and Assertion(i). Assertion (iv) follows by Assertion (i) and (iii), together with (35), (37) and (49).

Step 9. Assume that the negative-definite (respectively, positive-definite) high-gain property is known to hold. Steps $1-5$ of the above are unaffected by this assumption. Step 6 is readily modified as follows. By the assumption, there exists a positive (respectively, negative) real sequence $\left(s_{j}\right)$ such that the sequence $\left(\chi\left(s_{j}\right)\right)$ is unbounded, positive, and strictly increasing. Replacing $N$ by $s \mapsto s$ (respectively, by $s \mapsto-s$ ), the remaining arguments of Step 6 apply mutatis mutandis to conclude boundedness of $\alpha_{r}$. Steps 7 and 8 then follow as before. This completes the proof of the theorem.

Proof of Corollary 1.10 Let $y_{\text {ref }} \in W^{r, \infty}\left(\mathbb{R}_{\geq 0}, \mathbb{R}^{m}\right)$ and $y^{0} \in W^{r, \infty}\left([-h, 0], \mathbb{R}^{m}\right)$. By Theorem 1.9, the feedback-controlled system (1) and (9) has a solution, every solution can be maximally extended and every maximal solution is global. Let $y:[-h, \infty) \rightarrow$ $\mathbb{R}^{m}$ be any such global solution. In the following, we adopt the notation introduced in the proof of Theorem 1.9 and recall that, for all $k=1, \ldots, \hat{r}-1, \psi_{k}(\cdot)=0$, and for 
all $t \geq 0$,

$$
\begin{aligned}
\left\|e_{k}(t)\right\| & <1, \quad\left\|\gamma_{k}(t)\right\|=\alpha\left(\left\|e_{k}(t)\right\|^{2}\right)\left\|e_{k}(t)\right\|, \\
\left\|\dot{\gamma}_{k}(t)\right\| & =\left\|2 \alpha^{\prime}\left(\left\|e_{k}(t)\right\|^{2}\right)\left\langle e_{k}(t), \dot{e}_{k}(t)\right\rangle e_{k}(t)+\alpha\left(\left\|e_{k}(t)\right\|^{2}\right) \dot{e}_{k}(t)\right\| \\
& \leq \tilde{\alpha}\left(\left\|e_{k}(t)\right\|^{2}\right)\left\|\dot{e}_{k}(t)\right\| .
\end{aligned}
$$

Invoking (35), (37) and (40), with the convention that $\gamma_{0}(\cdot) \equiv 0 \equiv \dot{\gamma}_{0}(\cdot)$, we have, for almost all $t \geq 0$,

$$
\begin{aligned}
\left\|\dot{e}_{k}(t)\right\| & =\left\|(\dot{\varphi}(t) / \varphi(t))\left(e_{k}(t)-\gamma_{j-1}(t)\right)+e_{k+1}(t)+\dot{\gamma}_{k-1}(t)-\gamma_{k}(t)\right\| \\
& \leq M_{k}(t)+\left\|\gamma_{k}(t)\right\|, \\
\left\langle e_{k}(t), \dot{e}_{k}(t)\right\rangle & \leq M_{k}(t)-\alpha_{k}(t)\left\|e_{k}(t)\right\|^{2}, \\
M_{k}(t) & :=1+\mu_{0}\left(\left\|e_{k}(t)\right\|+\left\|\gamma_{k-1}(t)\right\|\right)+\left\|\dot{\gamma}_{k-1}(t)\right\| .
\end{aligned}
$$

Setting $k=1$, we have

$$
\left\langle e_{1}(t), \dot{e}_{1}(t)\right\rangle \leq \mu_{0}+1-\alpha_{1}(t)\left\|e_{1}(t)\right\|^{2} \text { for a.a. } t \geq 0 \text {. }
$$

With $e_{1}^{0}$ and $c_{1}$ as in (12), the argument used in Step 3 of the proof of Theorem 1.9 applies, mutatis mutandis, to conclude that $\left\|e_{1}(t)\right\| \leq c_{1}$ for all $t \geq 0$.

With $\mu_{1}=1+\mu_{0} c_{1}$ as in (12) we have, for almost all $t \geq 0$,

$$
\left\|\gamma_{1}(t)\right\| \leq c_{1} \alpha\left(c_{1}^{2}\right) \text { and }\left\|\dot{\gamma}_{1}(t)\right\| \leq \tilde{\alpha}\left(c_{1}^{2}\right)\left(\mu_{1}+c_{1} \alpha\left(c_{1}^{2}\right)\right)
$$

wherein we have used the facts that $\alpha$ and $\tilde{\alpha}$ are non-decreasing functions (monotonicity of the latter being assured by the assumption of monotonicity of $\alpha^{\prime}$ ). Now set $k=2$, in which case we have

$$
M_{2}(t) \leq 1+\mu_{0}\left(1+c_{1} \alpha\left(c_{1}^{2}\right)\right)+\tilde{\alpha}\left(c_{1}^{2}\right)\left(\mu_{1}+c_{1} \alpha\left(c_{1}^{2}\right)\right)=\mu_{2} \text { for a.a. } t \geq 0 .
$$

With $e_{2}^{0}$ and $c_{2}$ as in (12) the argument used in Step 4 of the proof of Theorem 1.9 applies, mutatis mutandis, to conclude that $\left\|e_{2}(t)\right\| \leq c_{2}$ for all $t \geq 0$. Iterating this process, we arrive at

$$
\forall k=1, \ldots, \hat{r}-1 \forall t \geq 0:\left\|e_{k}(t)\right\| \leq c_{k}
$$

To complete the proof, simply note that, for all $t \geq 0$,

$$
\begin{aligned}
& \varphi(t)\|e(t)\|=\left\|e_{1}(t)\right\| \leq c_{1} \quad \text { and } \\
& \varphi(t)\left\|e^{(k)}(t)\right\|=\left\|e_{k+1}(t)-\gamma_{k}(t)\right\| \leq c_{k+1}+c_{k} \alpha\left(c_{k}^{2}\right), \quad k=1, \ldots, \hat{r}-2 .
\end{aligned}
$$




\section{References}

1. Bechlioulis CP, Rovithakis GA (2008) Robust adaptive control of feedback linearizable MIMO nonlinear systems with prescribed performance. IEEE Trans Autom Control 53(9):2090-2099

2. Bechlioulis CP, Rovithakis GA (2014) A low-complexity global approximation-free control scheme with prescribed performance for unknown pure feedback systems. Automatica 50(4):1217-1226

3. Berger T (2014) Zero dynamics and stabilization for linear DAEs. In: Schöps S, Bartel A, Günther M, ter Maten EJW, Müller PC (eds) Progress in differential-algebraic equations, differential-algebraic equations forum. Springer, Berlin, pp 21-45

4. Berger T (2020) Funnel control of the Fokker-Planck equation for a multi-dimensional OrnsteinUhlenbeck process. arXiv:2005.13377v2

5. Berger T (2020) Tracking with prescribed performance for linear non-minimum phase systems. Automatica 115:108909

6. Berger T, Breiten T, Puche M, Reis T (2019) Funnel control for the monodomain equations with the FitzHugh-Nagumo model. arXiv:1912.01847

7. Berger T, Lanza L (2020) Output tracking for a non-minimum phase robotic manipulator. To appear in proceedings of the MTNS 2020. arXiv:2001.07535

8. Berger T, Lê HH, Reis T (2018) Funnel control for nonlinear systems with known strict relative degree. Automatica 87:345-357

9. Berger T, Otto S, Reis T, Seifried R (2019) Combined open-loop and funnel control for underactuated multibody systems. Nonlinear Dyn 95:1977-1998

10. Berger T, Puche M, Schwenninger FL (2020) Funnel control for a moving water tank. arXiv:1902.00586v2

11. Berger T, Puche M, Schwenninger FL (2020) Funnel control in the presence of infinite-dimensional internal dynamics. Syst Control Lett 139:104678

12. Berger T, Rauert AL (2018) A universal model-free and safe adaptive cruise control mechanism. In: Proceedings of the MTNS 2018. Hong Kong, pp 925-932

13. Berger T, Rauert AL (2020) Funnel cruise control. Automatica 119:109061

14. Berger T, Reis T (2014) Zero dynamics and funnel control for linear electrical circuits. J Franklin Inst 351(11):5099-5132

15. Berger T, Reis T (2018) Funnel control via funnel pre-compensator for minimum phase systems with relative degree two. IEEE Trans Autom Control 63(7):2264-2271

16. Berger T, Reis T (2018) The funnel pre-compensator. Int J Robust Nonlinear Control 28(16):4747-4771

17. Byrnes CI, Willems JC (1984) Adaptive stabilization of multivariable linear systems. In: Proceedings of the 23rd IEEE conference on decision control, pp 1574-1577

18. Ge SS, Hong F, Lee TH (2004) Adaptive neural control of nonlinear time-delay systems with unknown virtual control coefficients. IEEE Trans Syst Man Cybern B 34(1):499-516

19. Ge SS, Wang J (2002) Robust adaptive neural control for a class of perturbed strict feedback nonlinear systems. IEEE Trans Neural Netw 13(6):1409-1419

20. Ge SS, Wang J (2003) Robust adaptive tracking for time-varying uncertain nonlinear systems with unknown control coefficients. IEEE Trans Autom Control 48(8):1463-1469

21. Hackl CM (2014) (2014) Funnel control for wind turbine systems. In: Proceedings of IEEE international conference on control and applications, Antibes, France, pp 1377-1382

22. Hackl CM (2015) Current PI-funnel control with anti-windup for synchronous machines. In: Proceedings of the 54th IEEE conference on decision control, Osaka, Japan, pp 1997-2004

23. Hackl CM (2015) Speed funnel control with disturbance observer for wind turbine systems with elastic shaft. In: Proceedings of the 54th IEEE Conference on Decision Control, Osaka, Japan, pp 12005-2012

24. Hackl CM (2017) Non-identifier based adaptive control in mechatronics: theory and application, vol 466. Lecture notes in control and information sciences. Springer, Cham

25. Hackl CM, Hopfe N, Ilchmann A, Mueller M, Trenn S (2013) Funnel control for systems with relative degree two. SIAM J Control Optim 51(2):965-995

26. Huang J, Isidori A, Marconi L, Mischiati M, Sontag E, Wonham W (2018) Internal models in control, biology and neuroscience. In: Proceedings of the 57th IEEE conference on decision control, Miami Beach, FL, USA, pp 5370-5390

27. Ilchmann A, Ryan EP (2006) Asymptotic tracking with prescribed transient behaviour for linear systems. Int J Control 79(8):910-917 
28. Ilchmann A, Ryan EP (2009) Performance funnels and tracking control. Int J Control 82(10):18281840

29. Ilchmann A, Ryan EP, Sangwin CJ (2002) Systems of controlled functional differential equations and adaptive tracking. SIAM J Control Optim 40(6):1746-1764

30. Ilchmann A, Ryan EP, Sangwin CJ (2002) Tracking with prescribed transient behaviour. ESAIM Control Optim Calc Var 7:471-493

31. Ilchmann A, Ryan EP, Townsend P (2006) Tracking control with prescribed transient behaviour for systems of known relative degree. Syst Control Lett 55(5):396-406

32. Ilchmann A, Ryan EP, Townsend P (2007) Tracking with prescribed transient behavior for nonlinear systems of known relative degree. SIAM J Control Optim 46(1):210-230

33. Ilchmann A, Trenn S (2004) Input constrained funnel control with applications to chemical reactor models. Syst Control Lett 53(5):361-375

34. Ilchmann A, Wirth F (2013) On minimum phase. Automatisierungstechnik 12:805-817

35. Isidori A (1995) Nonlinear control systems, 3rd edn. Communications and control engineering series. Springer, Berlin

36. Jiang Z, Mareels IMY, Hill DJ, Huang J (2004) A unifying framework for global regulation via nonlinear output feedback: from ISS to iISS. IEEE Trans Autom Control 49:549-562

37. Lee JG, Trenn S (2019) Asymptotic tracking via funnel control. In: Proceedings of the 58th IEEE conference on decision control, Nice, France

38. Liberzon D, Trenn S (2013) The bang-bang funnel controller for uncertain nonlinear systems with arbitrary relative degree. IEEE Trans Autom Control 58(12):3126-3141

39. Logemann H, Mawby A (2000) Low-gain integral control of infinite dimensional regular linear systems subject to input hysteresis. In: Colonius F, Helmke U, Prätzel-Wolters D, Wirth FR (eds) Advances in mathematical systems theory. Birkhäuser, Boston, pp 255-293

40. Mareels IMY (1984) A simple selftuning controller for stably invertible systems. Syst Control Lett 4(1):5-16

41. Miller DE, Davison EJ (1991) An adaptive controller which provides an arbitrarily good transient and steady-state response. IEEE Trans Autom Control 36(1):68-81

42. Morse AS (1983) Recent problems in parameter adaptive control. In: Landau ID (ed) Outils et Modèles Mathématiques pour l'Automatique, l'Analyse de Systèmes et le Traitment du Signal, vol 3. Éditions du Centre National de la Recherche Scientifique (CNRS), Paris, pp 733-740

43. Na J (2013) Adaptive prescribed performance control of nonlinear systems with unknown dead zone. Int J Adapt Control Sign Process 27:426-446

44. Nussbaum RD (1983) Some remarks on a conjecture in adaptive control. Syst Control Lett 3:243-246

45. Pomprapa A, Alfocea SR, Göbel C, Misgeld BJ, Leonhardt S (2014) Funnel control for oxygenation during artificial ventilation therapy. In: Proceedings of the 19th IFAC world congress. Cape Town, South Africa, pp 6575-6580

46. Pomprapa A, Weyer S, Leonhardt S, Walter M, Misgeld B (2015) Periodic funnel-based control for peak inspiratory pressure. In: Proceedings of the 54th IEEE Conference on Decision Control, Osaka, Japan, pp 5617-5622

47. Puche M, Reis T, Schwenninger FL (2020) Funnel control for boundary control systems. Evol Equ Control Theory. https://doi.org/10.3934/eect.2020079

48. Reis T, Selig T (2015) Funnel control for the boundary controlled heat equation. SIAM J Control Optim 53(1):547-574

49. Ryan EP, Sangwin CJ (2001) Controlled functional differential equations and adaptive stabilization. Int J Control 74(1):77-90

50. Ryan EP, Sangwin CJ, Townsend P (2009) Controlled functional differential equations: approximate and exact asymptotic tracking with prescribed transient performance. ESAIM Control Optim Calc Var 15:745-762

51. Seifried R, Blajer W (2013) Analysis of servo-constraint problems for underactuated multibody systems. Mech Sci 4:113-129

52. Senfelds A, Paugurs A (2014) Electrical drive DC link power flow control with adaptive approach. In: Proceedings of the 55th international scientific conference on power and electrical engineering of Riga Technical University, Riga, Latvia, pp 30-33

53. Sontag ED, Wang Y (1995) On characterizations of the input-to-state stability property. Syst Control Lett 24(5):351-359

54. Spong MW, Hutchinson S, Vidyasagar M (2006) Robot modeling and control. Wiley, Hoboken 
55. Tao G, Kokotovic PV (1996) Adaptive control of systems with actuator and sensor nonlinearities. Wiley, New York

56. Tao G, Lewis FL (2001) Adaptive control of nonsmooth dynamic systems. Springer, London

57. Trentelman HL, Stoorvogel AA, Hautus MLJ (2001) Control theory for linear systems. Communications and control engineering. Springer, London

58. Twain M (1883) Life on the Mississippi. Osgood, Boston

59. Verginis CK, Dimarogonas DD (2019) Asymptotic stability of uncertain Lagrangian systems with prescribed transient response. In: Proceedings of the 58th IEEE Conference on Decision Control, Nice, France, pp 7037-7042

60. Verginis CK, Dimarogonas DD (2021) Asymptotic tracking of second-order nonsmooth feedback stabilizable unknown systems with prescribed transient response. IEEE Trans Autom Control. https:// doi.org/10.1109/TAC.2020.3015785

61. Wonham WM (1979) Linear multivariable control: a geometric approach, 2nd edn. Springer, Heidelberg

62. Ye X (2001) Adaptive nonlinear output-feedback control with unknown high-frequency gain sign. IEEE Trans Autom Control 46(1):112-115

Publisher's Note Springer Nature remains neutral with regard to jurisdictional claims in published maps and institutional affiliations. 